\title{
SEMI-AUTOMATIC PROSTATE SEGMENTATION OF MR IMAGES BASED ON FLOW ORIENTATION
}

\author{
By \\ Maryam Samiee \\ SUBMITTED IN PARTIAL FULFILLMENT OF THE \\ REQUIREMENT FOR THE DEGREE OF \\ MASTER OF SCIENCE \\ ELECTRICAL AND COMPUTER ENGINEERING \\ DEPARTMENT \\ UNIVERSITY OF MANITOBA \\ WINNIPEG, CANADA \\ DECEMBER 2006
}

(C) Copyright by Maryam Samiee, 2006

All rights reserved. This work may not be

reproduced in whole or in part, by photocopy or other means, without permission of the author. 
THE UNIVERSITY OF MANITOBA

FACULTY OF GRADUATE STUDIES

$* * * * *$

COPYRIGHT PERMISSION

\section{SEMI-AUTOMATIC PROSTATE SEGMENTATION OF MR IMAGES BASED ON FLOW ORIENTATION}

by

Maryam Samiee

A Thesis/Practicum submitted to the Faculty of Graduate Studies of The University of Manitoba in partial fulfillment of the requirement of the degree

of

Master of Science

Maryam Samiee (C) 2006

Permission has been granted to the Library of the University of Manitoba to lend or sell copies of this thesis/practicum, to the National Library of Canada to microfilm this thesis and to lend or sell copies of the film, and to University Microfilms Inc. to publish an abstract of this thesis/practicum.

This reproduction or copy of this thesis has been made available by authority of the copyright owner solely for the purpose of private study and research, and may only be reproduced and copied as permitted by copyright laws or with express written authorization from the copyright owner. 


\section{UNIVERSITY OF MANITOBA}

Date: December 2006

Author: $\quad$ Maryam Samiee

Title:

Semi-Automatic Prostate Segmentation of MR Images Based on Flow Orientation

Department: Electrical and Computer Engineering

Degree: M.Sc. Convocation: February Year: 2006

Permission is hereby granted to University of Manitoba to circulate and to have copied for non-commercial purposes, at its discretion, the above title upon request of individuals or institutions.

Signature of Author

THE AUTHOR RESERVES OTHER PUBLICATION RIGHTS, AND NEITHER THE

THESIS NOR EXTENSIVE EXTRACTS FROM IT MAY BE PRINTED OR

OTHERWISE REPRODUCED WITHOUT THE AUTHOR'S WRITTEN

PERMISSION.

THE AUTHOR ATTESTS THAT PERMISSIONS HAS BEEN OBTAINED FOR THE USE OF ANY COPYRIGHTED MATERIAL APPEARING IN THIS THESIS (OTHER THAN BRIEF EXCERPTS REQUIRING ONLY PROPER ACKNOWLEDGMENT IN SCHOLARY WRITING) AND THAT ALL SUCH USE IS CLEARLY ACKNOWLEDGED. 
Ta My Family,

Mahammad Reza and Mahyar 


\section{ACKNOWLEDGEMENT:}

I would like to take this opportunity to thank those people who have helped me along the way.

First and formest, I would like to thank my advisor Dr. Gabriel Thomas. His wealth of ideas, enthusiasm and energy has made working with him an unforgettable experience for me. I am also thankful to Dr. Reza Fazel-Rezai and Dr. Boyd McCurdy for being in my defence and reading committee. I would like to especially acknowledge Niranjan Venugopal, for his collaboration on this research and providing much valuable feedback.

My heartfelt acknowledgement goes to my teachers in all the stages of my education, the individuals who have inspired me to learn and grow. I especially would like to thank, Mr. Gholamreza Nasri and Mr. Bahram Pourali, my undergrad supervisors for their valuable support and encouragement in KNTU and ITRC in Iran.

I also give my gratitude to my fellow graduate students. In particular, I would like to thank Azadeh, Ali, Arash and January for their provoking questions and commands on my work. I especially like to thank Aimee Betker for her through reading of the draft of this thesis and her efficient assistance and helpful attitude.

My deepest love and gratitude belong to my entire family, my parents, brothers, husband and son, for their unconditional love and support. This thesis is dedicated to my husband Mohammad Reza and my son Mahyar. 


\section{ABSTRACT}

Prostate cancer is a leading cause of death in men. Magnetic Resonance Spectroscopic Imaging (MRSI) has been demonstrated to be beneficial for the diagnosis, staging and past treatment follow-up of prostate cancer. During the MRSI acquisition, segmentation of the prostate images is required, in order to allow the maximum volume of the prostate to be considered while reducing signal contribution from outside the prostate which may impact accuracy of the MRSI. Manual segmentation is a time consuming job; hence, a semi-automatic method was investigated, which segments the prostate on the magnetic resonance images acquired in the same imaging session as the MRS images. The algorithm evaluates the curve of the prostate boundary in an orientation-based framework. Once the directional field of the pixels in the prostate region is calculated, a statistical average mask follows the direction of low-density pixels around the prostate, using prior knowledge of the prostate's shape. This knowledge is obtained through user selection of four points in the prostate region along with the prior knowledge of the shape of the prostate and its neighbouring organs. Compared to manual segmentation by an expert, the initial results of our algorithm are promising. Currently, accurate segmentation has not yet been achieved and the development of a completely automatic and accurate method remains an interesting research topic. 


\section{TABLE OF CONTENTS}

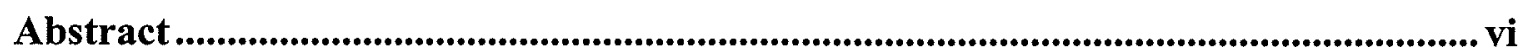

Table of Contents ...................................................................................................... vii

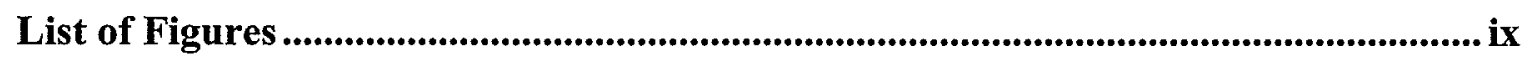

1 Introduction and Objective .......................................................................................... 1

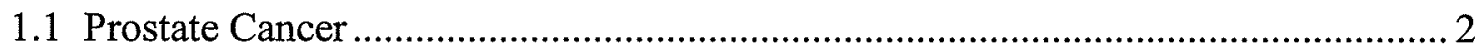

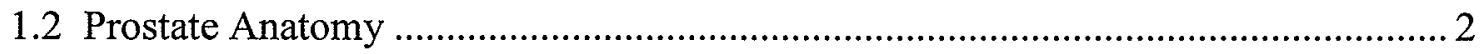

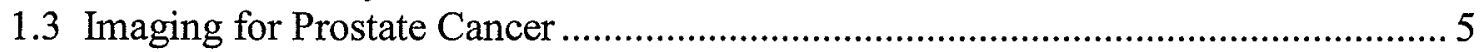

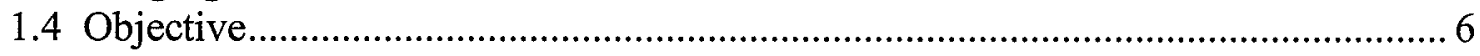

1.5 Literature Review ............................................................................................

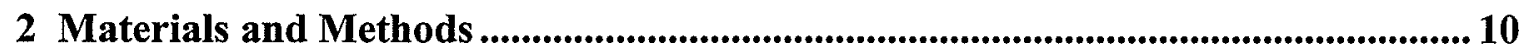

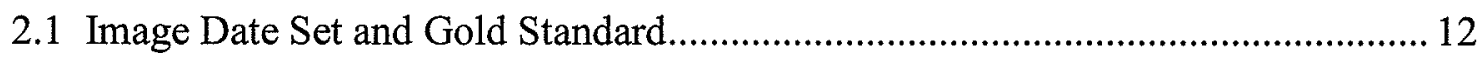

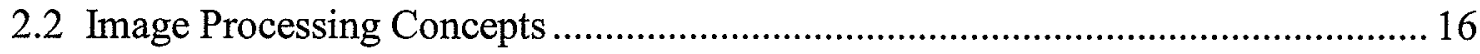

2.2.1 Ridgeline Tracing Concepts .................................................................. 19

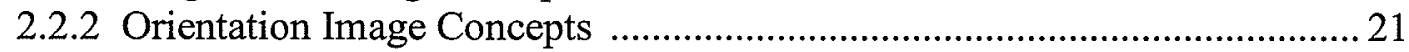

2.2.3 Shape Model Concepts …………............................................................... 23

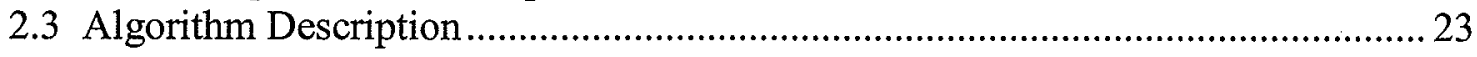

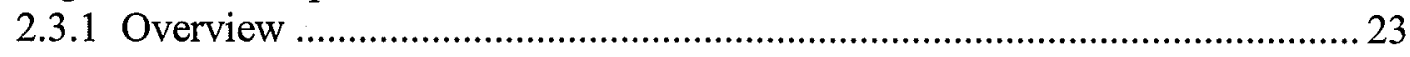

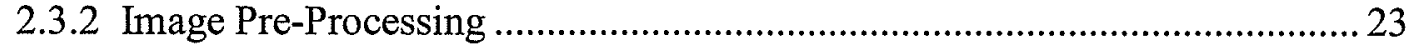

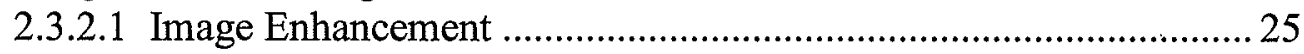

2.3.2.2 Statistical Normalization ................................................................ 27

2.3.2.3 Orientation Image Calculaton.......................................................... 29

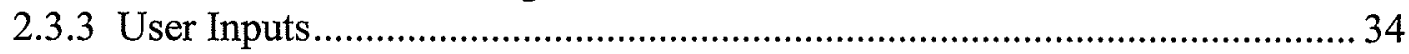

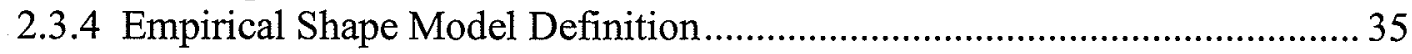

2.3.5 Boundary Direction Estimation ..................................................................35

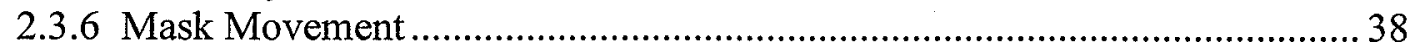

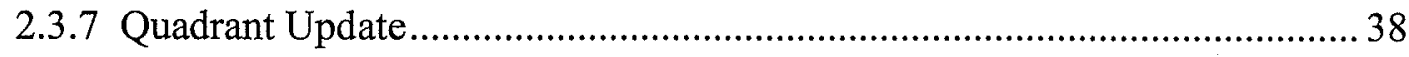

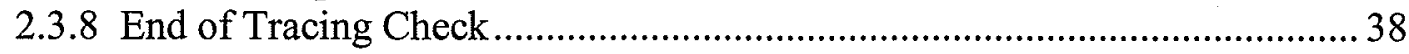

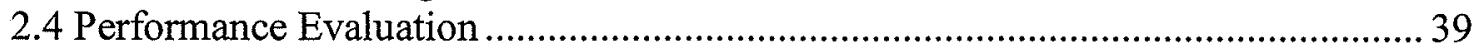

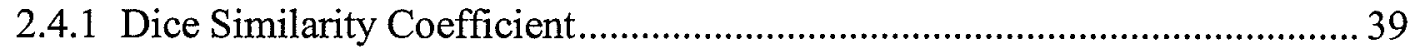

2.4.2 Mean and Variance Analysis ...................................................................... 42

2.4.3 Statistical Ranking Analysis ....................................................................... 43 


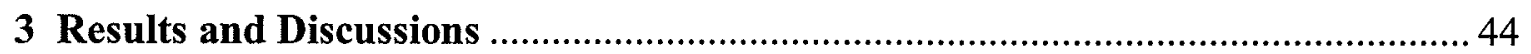

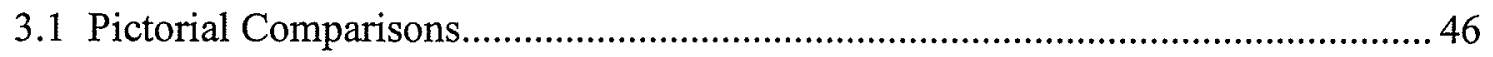

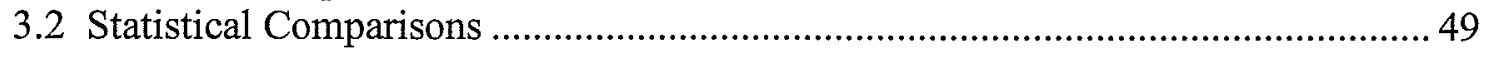

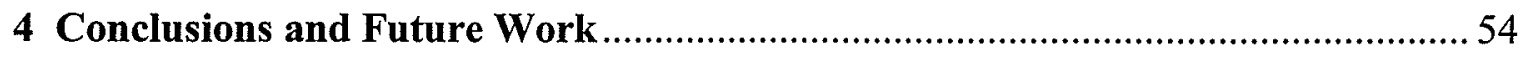

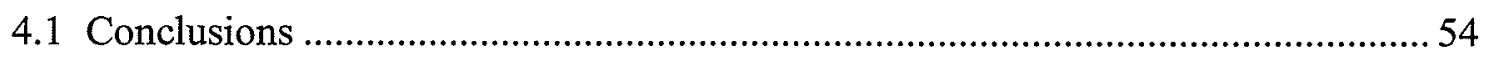

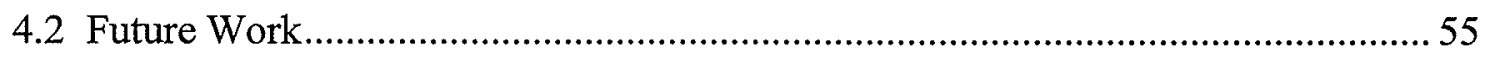

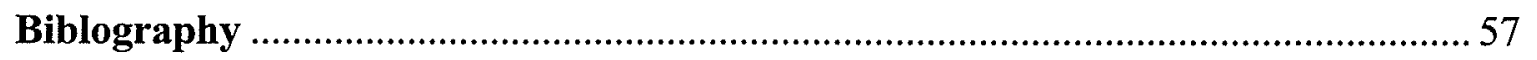




\section{LIST OF FIGURES}

Figure 1-1 Prostate gland and extended tumour .............................................. 4

Figure 2-1 Two typical images of the MR prostate data set: (a) slice number 10 of the inflated set; and (b) slice number 10 of the deflated data set.

Figure 2-2 Two typical images of the MR prostate gold standard data set: (a) slice number 10 of the inflated set; and (b) slice number 10 of the deflated set

Figure 2-3 Direct grey-scale ridge tracing in fingerprint: (a) fingerprint grey scale image with concentration on the ridges (dark areas) and valleys (light areas); and (b) ridgeline following steps. Reprinted with permission from Springer-Verlag [11]

Figure 2-4 A pictorial comparison between orientation images of: (a) a fingerprint; and (b) a prostate MRI with inflated endorectal coil (T2weighted). Small bars in the image represent the orientation of the ridge-valley structures

Figure 2-5 A surface S, corresponding to a small area of the fingerprint. Reprinted with permission from Springer-Verlag [11]

Figure 2-6 Detailed area in a fingerprint. (a) the gradient; (b) and the average data field. Reprinted with permission from A. M. Bazen [12]

Figure 2-7 General flowchart of the algorithm

Figure 2-8 Histograms of the enhancing process. (a1) original image histogram; (b1) inverted image histogram; (c1) normalized histogram of noninverted image; and (d1) normalized histogram of inverted image.

Figure 2-9 Images of MR prostate enhancing process: (a1) original image; (b1) inverted image; (c1) normalized non-inverted image; and (d1) normalized inverted image

Figure 2-10 Images and histograms for: (a1)(b1)enhanced; (a2) (b2) general statistical normalization 
Figure 2-11 General block diagram of the orientation image calculation

Figure 2-12 Images showing the effects of the smoothing filters after non-linear operators and their size effect: (a) no smoothing filter; (b) filter size 19 and $\mathrm{STD}=3$; (c) filter size 25 and $\mathrm{STD}=4$; (d) filter size 31 and $\mathrm{STD}=5$.

Figure 2-13 Images showing different steps of orientation image computation, assuming $G$ is the image block levels of size $7 \times 7$ : (a1) $G_{\mathrm{X}}$; (a2) $G_{\mathrm{Y}}$;(b1) $G_{\mathrm{XX}}$; (b2) $G_{\mathrm{YY}}$; (b3) $G_{\mathrm{XY}}$; (c1) gradient directions in block level; and (c2) directional field in block level.....

Figure 2-14 Estimation of valid boundary area which uses defined points shown on $\mathrm{A}, \mathrm{B}, \mathrm{C}, \mathrm{D}$ and quadrants $1,2,3,4$

Figure 2-15 Empirical shape model of prostate 35

Figure 2-16 Estimation of boundary direction: (a) definition of quadrants, initial points and centre of mass; (b) the average of the pixel field direction covered by the mask is used for calculating the new position; (c) emphasizing the concept of orientation without direction; (d) a look up table showing the orientation patterns based on the quadrant location

Figure 2-17 The completion of the tracing procedure: the algorithm continues to trace the boundary until the tracer moves into the neighbourhood of starting point in quadrant four: (a) end of tracing in quadrant 3 ; (b) tracing in quadrant 4 ; (c)end of tracing in quadrant 4 or end of tracing

Figure 2-18 DSC calculating procedure: The above flowchart indicates the procedure for calculating DSC using the Gold Standards and the results of suggested semi-automatic segmentation method 41

Figure 3-1 Pictorial show of DCS components for performance evaluation. (a) filled contour of gold standard. (b) filled contour of segmented prostate. (c) area of coincidence. (d) area of prostate segmented as background; and (e) area of background segmented as prostate. Images correspond to the inflated set, slice number 10 . 
Figure 3-2 Comparison of manual (red) and semi-automatic (blue) segmentation. Images correspond to the inflated endorectal coil case

Figure 3-3 Comparison of manual (red) and semi-automatic (blue) segmentation. Images correspond to the deflated endorectal coil

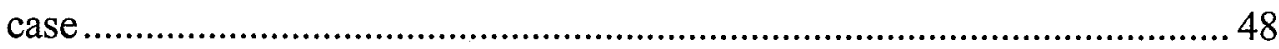

Figure 3-4 Statistical analysis of DSC for the deflated set, using mean-variance .......51

Figure 3-5 Statistical analysis of DSC for the inflated set using mean-variance ........ 52

Figure 3-6 Statistical analysis of DSC using box plots, for both inflated and deflated set. 


\section{CHAPTER 1}

\section{Introduction and Objectives}

Concerning cancer related illnesses in males, prostate cancer is the second leading causes of death [1]. Magnetic Resonance Imaging (MRI) is one of the primary choices for the diagnosis, staging and follow-up of prostate disease. Accurate segmentation of prostate magnetic images is required, in order to allow the maximum volume of the prostate to be considered in both diagnosis and treatment. This work presents a semiautomatic method, which segments the prostate magnetic resonance images. Due to poor image contrast, low signal to noise ration (SNR) of the acquisition system and many other reasons, reconstructing a geometric representation of prostate on MR Images is a difficult task; to date, neither an accurate segmentation nor the development of an automatic method has been achieved.

This work is organized as follows. In chapter 1 prostate anatomy, cancer and imaging techniques, the objectives and the literature background are discussed. Chapter 2 introduces the data set and describes the research methodology in detail, including the general algorithm, important functions and a comprehensive discussion concerning the orientation image. Chapter 3 presents the results and discussions. Lastly, in Chapter 4 the conclusions and the future work are discussed. 


\subsection{Prostate Anatomy}

The prostate gland forms part of the male reproductive system. One of the most important functions of this gland is to secret a slightly alkaline fluid that is found in male seminal fluid. The prostate is located in front of the rectum surrounding the neck of the bladder and urethra (the tube that carries urine from the bladder). The gland is made up of three lobes (a central lobe with an adjacent lobe on each side) which are composed of partly muscular and partly glandular tissue with ducts opening into the prostatic of the urethra.

\subsection{Prostate Cancer}

Prostate cancer is the second leading cause of cancer related deaths in males. It constitutes approximately $27 \%$ of all male cancers and it is the cause of $13 \%$ of male cancer related deaths [1].

Surveys conducted in western populations show that the occurrence of prostate cancer has increased noticeably over the past 35 years; hence, the diagnosis and supervision of prostate cancer have become important health concerns. Currently, an average of 13500 new prostate cancers are diagnosed each year in the UK [2]. In Canada, this number has been estimated at 20700 per year [55].

In general, cancer is thought to be a result of damage to genes that control cell reproduction. Normal somatic cells undergo a predictable cycle of growth and renewal; as old cells die off, new cells form to replace them. When genes become damaged, cells may begin to grow and reproduce in an uncontrolled manner. These cells are cancerous and can occur is many tissues in the body. Prostate cancer is a group of these abnormal 
cells localized in the male prostate. Prostate cancer may spread via the blood supply or the lymphatic system. If prostate cancer spreads distantly beyond the prostate gland, metastasis is said to occur.

While the cause of prostate cancer remains unknown, several risk factors have been identified. These include uncontrolled factors such as age, race and family history, as well as controllable factors such as diet.

Stage, grade and pre-treatment prostate specific antigens (PSA) are the three most important prognostic indicators [53]. PSA's are produced by the prostate and released into the blood. The levels of PSA tend to increase in prostate cancer patients. To define which treatment modality is best and for whom, adequate information is required.

Clinical staging of the primary tumour is determined by digital rectal examination along with imaging studies [53]. Digital rectal examination and transrectal ultrasound, as an imaging modality are poor in predicting disease extent at the surgery time [53]. Therefore, there has been intensive interest in providing more precise staging information considering more complicated imaging techniques.

The utility of a staging $C T$ scan is limited in most patients in the early clinical stage with the low risk features [53].

Recent improvement in $M R I$ have shown promise in improving clinical staging, especially in predicting local extension of disease beyond the gland by using endorectal coil. These studies have presented remarkable outcomes in low to intermediate risk patients [53]. 
Due to the relative inaccuracy of clinical staging using digital examination and available imaging modalities, histogram tumour grade and pre-treatment PSA levels are valuable auxiliary techniques by which local tumour extension can be assessed [53].

In the developed cases, radio isotopic bone scan has been advocated as part of the staging, because the skeleton is the most common distant metastatic site for prostate cancer [53].

There are many different methods for treating cancer as well. Radical prostatectomy with bilateral pelvic lymph node dissection might be appropriate for patients diagnosed with an organ-restricted disease [2]. However, once the tumour has extended behind the gland (Figure 1.1), radical radiotherapy becomes the best option. For developed cases, hormone deprivation, radiotherapy and chemotherapy can all be important treatments.

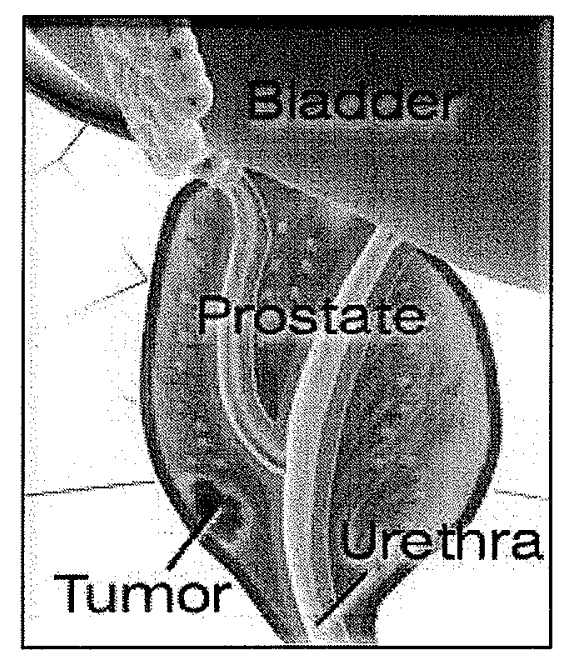

Fig.1.1. Prostate gland and extended tumour. 


\subsection{Imaging for Prostate Cancer}

As mentioned in the previous subsection, different non-invasive methods of imaging are available in order to diagnose, follow up and treat prostate cancer, these methods include: Ultrasound, $C T$ and MR Imaging.

Ultra sound Imaging, also called ultrasound scanning or sonography, involves exposing prostate to high-frequency sound waves to produce pictures of the gland. Ultra sound exams do not use ionizing radiation (x-ray). An advantage of ultrasound imaging is that the images are captured in real-time and therefore immediately show the structure of the target tissue. Ultrasound imaging is usually a painless medical test that helps physicians diagnose and treat medical conditions.

As part of the diagnosis process, prostate cancer patients can be expected to undergo additional tests to determine if the cancer has spread beyond the prostate. These tests may include a CT scan. CT scan, also called CAT scan, stands for Computerized Axial Tomography. A machine revolves around the patient, generating a series of images that are then translated by a computer. These images contain anatomical cross-sections of the prostate [57].

Magnetic resonance imaging (MRI) is another volumetric form of imaging, which provides an accurate non-invasive way of viewing soft tissues like prostate without the use of $\mathrm{x}$-rays. MRI uses a magnetic field and radio waves together with a computer to create cross-sectional pictures of prostate. Detailed MR images allow physicians to better evaluate parts of the body that may not be visible with other imaging methods such as $\mathrm{x}$ ray, ultrasound or computed tomography (CT). MRI has proven valuable in diagnosing a broad range of conditions, including cancer [57]. 
MR Images of the prostate exhibit excellent soft tissue contrast, but they suffer from distortion and slice thickness error [4]. Distortion may change the relationship between a displayed voxal and the real part of the patients' body. Therefore, MR Images are typically not the exclusive source of information.

In most individuals, prostate cancer is not detectable by routine imaging modalities such as ultrasound, CT and MRI. It has been shown that MRI combined with proton MRSI as a complementary technique, might be more suitable for examination of anatomical and metabolic processes of tumours and provide better information about the location and spatial extent of cancers within the peripheral zone of the prostate gland [55]. Standardized proton magnetic spectroscopic imaging was developed for routine assessment of human brain tumours initially. It was extended for examination of prostate and breast cancer later [55].

\subsection{Objectives}

MRI provides 3D anatomical information of the prostate, which is commonly displayed as $2 \mathrm{D}$ transverse for axial slices. The main goal of this research is to investigate these $2 \mathrm{D}$ axial images and segment the prostate organ. Instead of traditional manual segmentation, the goal is to develop a semi-automatic method to delineate the prostate boundary for each of the slices, in a fast and accurate way.

Medical image segmentation algorithms are faced with difficult challenges; for example, poor image contrast due to missing or diffused boundaries because of blending with the surrounding tissues, patient movement, low SNR of the acquisition system and many other reasons. The shape and signal intensity pattern of the prostate can vary with 
time, as cancer develops, and between individuals [2]. Under these difficult conditions and without having a prior model to constrain the segmentation, most algorithms are prone to errors [22].

The algorithm presented here is based on orientation information obtained from the MRI, which is used to calculate the direction of a tracing curve. The location of four points within the boundary of prostate are defined by the user a priori. This information is used together with the prior knowledge of the object shape in order to define the trajectory of a $3 \times 3$ mask. The mask will be used to trace the channel around the prostate gland. This is a semi-automatic method for segmentation of the prostate MRI.

\subsection{Literature Review}

In general, medical image segmentation methods can be divided into two basic groups:

1) Non model-based methods, which do not imply any pre-knowledge of the object.

2) Model-based methods, which consider an object shape model, appearance model or a combination of these two.

An example of the first group is the Kass approach, which employs a deformable contour in order to fit the shape of interest in the image [4]. This algorithm constitutes a general technique of matching a deformable model onto an image by means of energy minimization within a $2 \mathrm{D}$ or $3 \mathrm{D}$ image. However, most of the recent approaches are based on the model-based methods [8]; more specifically, a shape-based model. In [5], a shape-based approach for segmentation of medical images with a known object type in 
3D is proposed. In order to evaluate the curve, a parametric model was derived for an implicit representation of the segmentation curve, as a geometric active contour. In this way, active contours (explained earlier in this subsection) are represented implicitly as level set of functions defined on higher dimensional manifolds in a Eurelian formulation [59]. These models have been extracted using a collection of signed distance representations of the training data. This method has been applied to two medical applications [5], one of which is 3D segmentation of prostate MRI. Pictorial results show, working in 3D space, utilizes the full $3 D$ structural information of the prostate for segmentation and does not display any of the "step-like" artefacts that mar the radiologist's 3D rendition of the prostate gland. However, there is no quantitave comparison available. Dryden and Mardia [6] described statistical models of shape. Ladek [7] used a model-based Discreet Dynamic Contour (DDC) for prostate segmentation from ultrasound images.

In a recent approach, Freedman uses both the shape and appearance of objects (pixel intensity) in a model-based approach, in order to achieve segmentation [8]. Since this more complete approach combines both shape and appearance models, it can overcome some challenges specific to medical images, such as objects of the same shape with similar intensity profile, and interest and non-interest in a tiny area of the image. The pictorial results for a couple of slices are promising. However, there is no statistical analysis of the outcomes included.

Another interesting and recent approach by Zwiggelaar employs a semi-automatic polar transform method (starting point somewhere inside the prostate), using scale space ridge detection. As the learned shape of the prostate on the $2 \mathrm{D}$ axial slices are assumed to 
be circles, this method is classified as a model-based method [1]. Other simple techniques like line detection and non-maximum suppression are used to track the boundary of the prostate in the above method. Accuracy analysis of the results is promising for a limited group of the middle slices.

Our novel method can be considered a 2D model-based method, which considers a predefined shape for the prostate, a valid area of search and that the 4 starting points are in the boundary; hence, the object of interest can be traced and extracted. 


\section{CHAPTER 2}

\section{Materials and Methods}

\subsection{Image Data Set and Gold Standard}

Our data set consisted of two MRI volume sets, each one containing 19 axial slices. The first volume set was obtained using an inflated endorectal coil, which will be referred to as the inflated set; the second set was obtained using a deflated endorectal coil, which will be referred as the deflated set. The deflated set is not clinically common used except in deformation studies. The purpose of using inflated endorectal coil is to help establish evidence of extra-capsular extension in prostate cancer, when it incorporates magnetic resonance imaging. The endorectal coil is a receive-only coil, which is mounted inside a latex balloon; once inflated in the patient's rectum, its diameter is about $4-6 \mathrm{~cm}$. Filling the coil with a certain amount of air is called inflating. In this clinical experiment, the coil is filled with approximately $100 \mathrm{cc}$ of air. This enables a more detailed image of the prostate gland to be taken by improving SNR and providing sufficient image contrast. However, using the coil deforms the prostate by pushing it superiorly and anteriorly.

All images were obtained on a 1.5 Tesla magnet (Genesis-Signa, GE Medical Systems, Milwaukee, USA) using an endorectal coil. The field of view was $14 \times 14 \mathrm{~cm}$, 
the image size was $512 \times 512$ pixels, the slice thickness was $3.5 \mathrm{~mm}$ with an inter-slice gap of $3.9 \mathrm{~mm}$, the TR (Repetition Time) was $3600 \mathrm{~ms}$, and the TE (Echo Time) was 106 ms with the resolution 3.657 pixel per squared millimetres. Figure 2.1 shows two typical examples from the data set. In both cases, the prostate can be found in the centre of the image. Typical acquisition times were 5-6 minutes. The patient was placed in a supine position for the imaging examination. We propose to design a semi-automatic method, which would help the radiation oncologist to extract the boundary of the prostate for both data sets, in a more efficient manner.

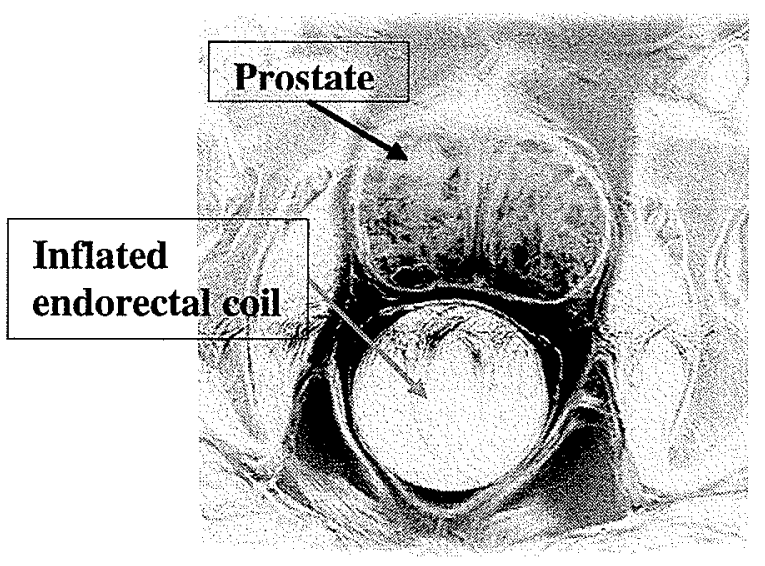

(a)

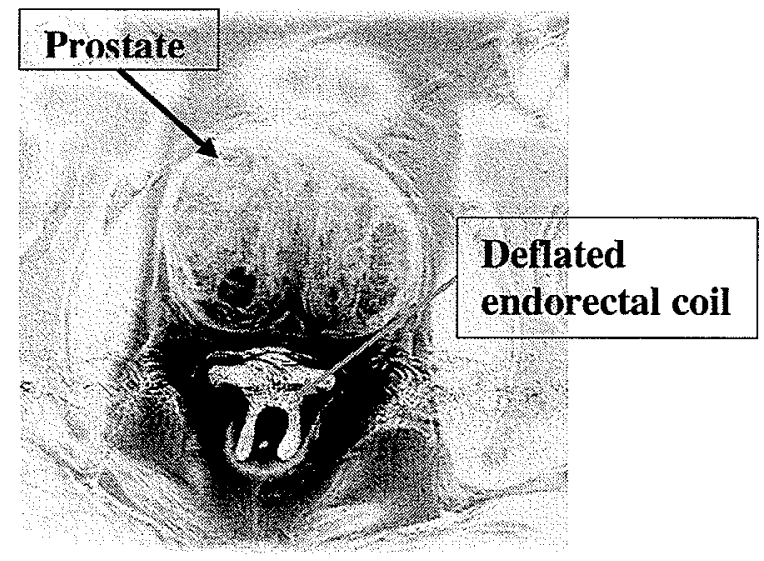

(b)

Fig. 2.1. Two typical images from the MR prostate data set: (a) slice number 10 of the inflated data set; and (b) slice number 10 of the deflated data set.

In order to obtain a "Gold Standard" data set to validate our methods, a single expert radiation oncologist manually contoured the prostate in the two MRI data sets, 10 times with the contouring sessions separated by some time delay. The average DSC (it is a metric of accuracy that is described in subsection (2.4.1)) of the Gold Standard data set 
were 0.913 (inflated) and 0.924 (deflated) respectively [39]. These two numbers indicate the intra-operator accuracy that shows the limit of accuracy of the Gold Standard by itself. After post-processing the annotated results, the Gold Standards take the form of binary images, as illustrated in Figure 2.2; note that these Gold Standard images correspond to the images in Figure 2.1.

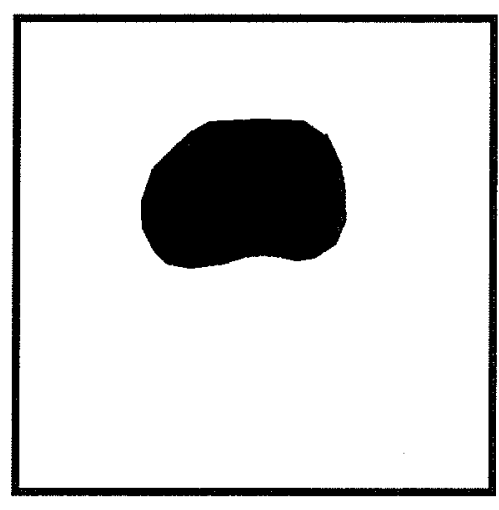

(a)

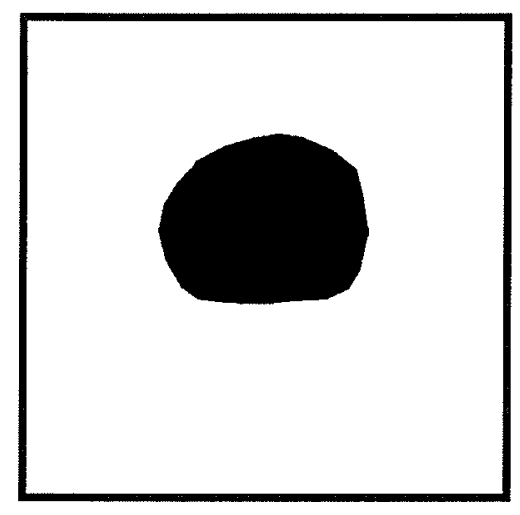

(b)

Fig. 2.2. Two typical images from the MR prostate Gold Standard data set: (a) slice number 10 of the inflated data set; and (b) slice number 10 of the deflated data set.

\subsection{Image Processing Concepts}

The proposed segmentation algorithm is based on two important ideas: ridgeline tracing, orientation image, and the shape model. The main idea is ridgeline tracing: after a user initializes the four starting points, the algorithm is able to trace the prostate boundary (i.e. the ridge curve in the original MR Image) without any more user interactions. This concept was inspired by research done by $\mathrm{D}$. Maio on fingerprint classification [9]. Two important definitions were used in [9]: the gradient direction and the local maxima of the grey scale fingerprint image, which allowed tracing the direction of the ridges for minutiae detection. 
In order to define the starting points, a square-mesh grid is superimposed on the image, each one defining a starting point in the dark area. After choosing the starting point $\left(i_{c}, j_{c}\right)$, the algorithm finds the gradient direction $\phi_{c}$ for the current point. In the length $2 \sigma+1$ neighbourhood of $\left(i_{c}, j_{c}\right)$ contained in the intersection of perpendicular plane at the gradient direction in the spot, the local maxima are then estimated. There is usually more than one local maximum; the maximum that is closest to the previous detected direction is the one selected for the next movement. By moving $\mu$ pixels from $\left(i_{c}, j_{c}\right)$ along the direction $\phi_{c}$ at each step, a new point $\left(i_{t}, j_{t}\right)$ is computed. The algorithm keeps following the ridgelines until they intersect other ridgelines or terminate. Eventually a labelling strategy is used to examine each ridgeline only once and the intersection between ridgelines is located. Depending on the average thickness of the ridgelines, the parameters $\mu$ and $\sigma$ can be optimized. An overview of how this method works is depicted in Figure 2.3.

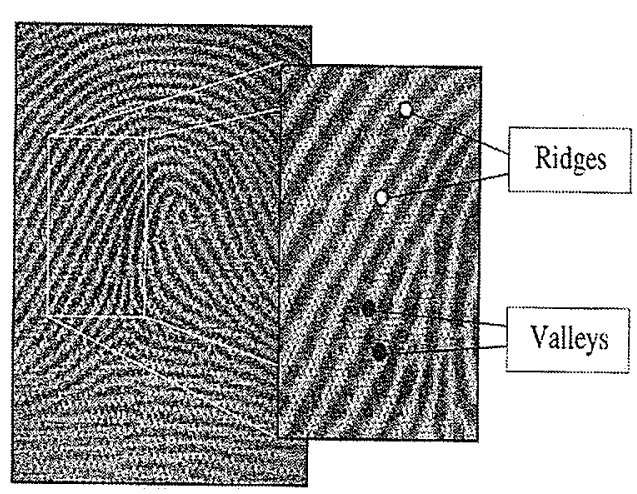

(a)

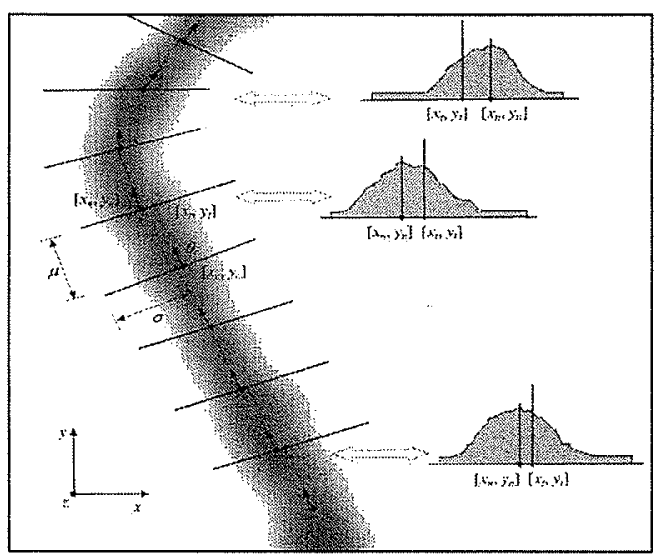

(b)

Fig. 2.3. Direct grey-scale ridge tracing for a fingerprint: (a) fingerprint grey scale image with concentration on the ridges (dark areas) and valleys (light areas); and (b) ridgeline following steps. Reprinted with permission from Springer-Verlag [11]. 
According to the above explanation, the tracings obtained using the local maxima define the ridge. Finally, by connecting these maxima in order, a ridgeline is obtained that is approximated by a polygon. Comparing the prostate boundary with the fingerprint image motivates the idea that the boundary of the prostate can be considered as a locally oriented pattern (Figure 2.4).

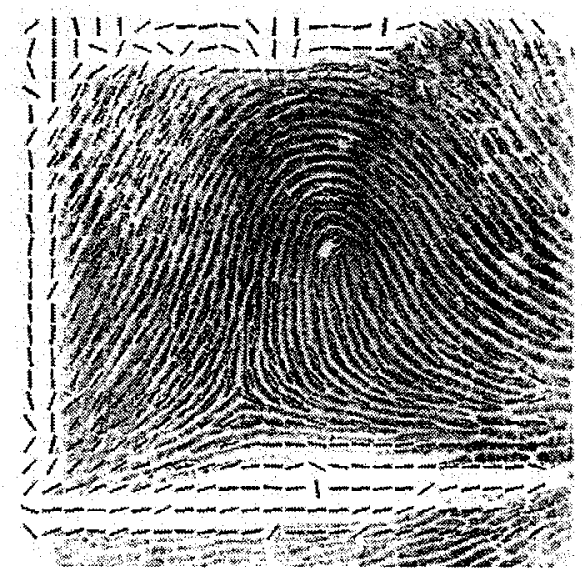

(a)

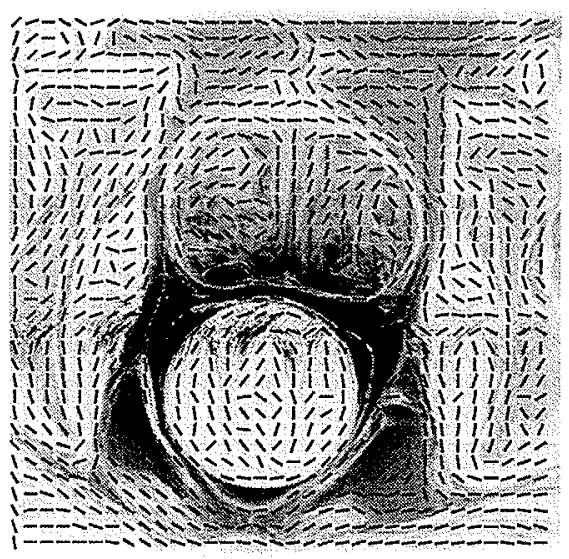

(b)

Fig.2.4. A pictorial comparison between the orientation images of: (a) a fingerprint; and (b) a prostate MRI with inflated endorectal coil (T2-weighted). Small bars in the above image represent the orientation of ridge-valley structures.

However, the ridge patterns in the grey level image of a fingerprint comparing with the boundary of the prostate are more complicated. The ridge patterns in a fingerprint image are almost isolated from each other, with the minima of the edge patterns located in between. Conversely, the boundary of the prostate is a thick ridge area, with a number of tiny ridge patterns surrounding it; these ridge patterns originate from both inside and outside the prostate. In addition, the thickness of the boundary around the prostate is not consistent, which makes choosing $\mu$ and $\sigma$ an adaptive 
problem. Lastly, this method of tracing is very pixel-based and noise-sensitive. Considering these issues, the method of detecting the direction used in tracing fingerprints is not appropriate for tracing the boundary of the prostate; therefore, a coarser method is needed to estimate the boundary curve direction in prostate MRI. As well, an orientation image, which aids in structure direction estimation, can be used instead of finding the maxima directions.

The orientation image for a fingerprint, first introduced by Grasselli [10], is a two-dimensional $(2 D)$ matrix. Each element: a) encodes the local orientation of the fingerprint ridges; b) corresponds to one node in a square-meshed grid, which is located over all pixels; and c) denotes the average orientation of the fingerprint ridges, in the neighbourhood of an individual pixel [11].

Once the starting points are initialized, the local tracing of the boundary can be performed in a straightforward manner by computing the orientation image, also known as the directional field $(D F)$, of the MR images. The orientation image is an inherent property of the image and it is the second important idea that our algorithm is based on; it can help us to provide estimations for the direction of the ridges and valleys in the image and it is described in more details in subsection (2.2.2). Therefore, using averaging and moving windows will allow for extraction of a coarse direction identifier of the ridges and valleys. However, the surrounding organs could still be problematic for the tracer; thus, constraints must be identified which will prevent the tracer from potential errors due to these distracting factors. One approach is to define correct and incorrect curve directions in the different areas of the prostate boundary. In the proposed segmentation algorithm, based on the location of four initial inputs, four different quadrants of the 
boundary are defined. According to the specific shape of the curve in each quadrant and the fact that the shapes in each volume set follow a similar curve change pattern, an estimation of the valid curve direction corresponding to each quadrant can be evaluated. These concepts are briefly explained in subsection (2.2.3) where the shape model concepts as the last important idea are discussed.

\subsubsection{Ridgeline Tracing Concepts}

As previously mentioned, our algorithm is founded on two important concepts: ridgeline tracing and the use of an orientation image. The prostate boundary is determined by following the ridgelines of interest in the grey scale axial image of the prostate; this tracing is based on the orientation image (or $D F$ ) of the prostate MR.

In order to define the ridgelines, we first obtain $I$, a $2 D$ grey level image, where

$I(i, j)$ indicates the grey level of pixel $(i, j)$, with $i=1, \ldots a$ and $j=1, \ldots b$. Bright pixels are associated with grey levels near zero and dark pixels are associated with grey levels near $g-1$, where $g$ denotes the number of grey levels in the image. Then, the prostate ridgelines correspond to surface ridges and the spaces between the ridgelines correspond to surface edges or valleys (Figure 2.5). 


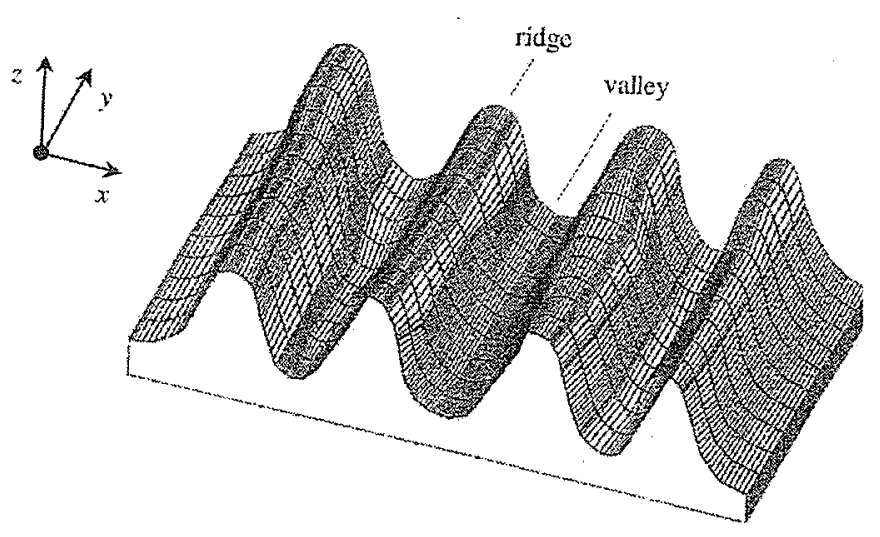

Fig. 2.5. A surface $S$, corresponding to a small area of the fingerprint. Reprint with permission from Spring-Verlag [11].

Note that gradients provide the same information as orientation images do, but at the pixel level; the orientation image describes the orientation of the ridge-valley structures. During computation of the orientation image, a region of pixels is covered. Hence, the resulting information is not pixel based; it is a coarse scale comparing gradient information. Although not all of the gradients in individual pixels are parallel, the resulting orientation is parallel, since the averaging operators are to calculate the orientation of these partial areas (Figure 2.6) [13].

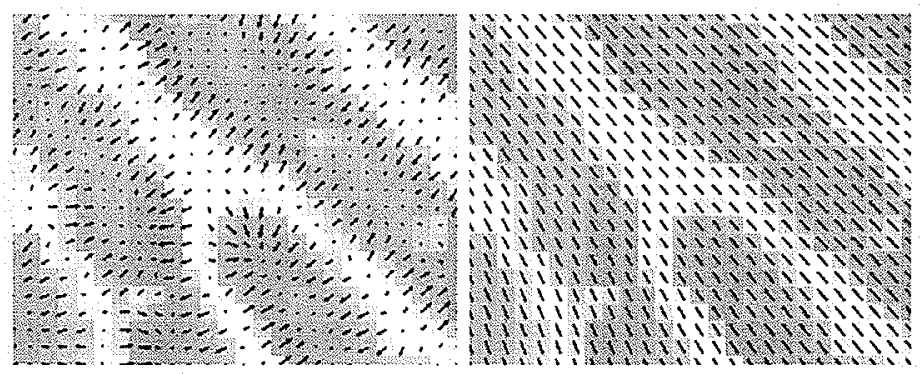

(a)

(b)

Fig. 2.6. Detailed area in a fingerprint: (a) the gradient; and (b) the average directional field. Reprint with permission from A. M. Bazen [12]. Arrows in (a) represents pixel gradient direction while bars in (b) represents ridge-valley orientation. 
In theory, once the averaging operations are performed on the individual pixel gradients in a given neighbourhood, we should be able to extract the orientation image; however, this is not always the case. In some local neighbourhoods, opposite gradient vectors cancel each other out, even though they indicate the same ridge-valley orientation. This happens when the ridge-valley structures rotate over 180 degrees [13]; in this case, they remain unchanged. Since the gradient orientations are distributed over a periodic space from zero to $\pi$, another method of determining the average orientation is by computing the " $\pi$-periodic cyclic mean."

According to [14], a solution to this problem can be obtained by doubling the gradient vector angles prior to averaging them. Afterwards, opposite gradient vectors will indicate the same direction and will therefore augment each other; nevertheless, perpendicular gradients will still cancel each other out. All of these steps must be considered when computing the orientation image as will be described in subsection (2.3.2.3).

\subsubsection{Orientation Image Concepts}

Several methods for computing the orientation image are proposed in the literature; for example, match filter approaches were used in [15], [17] and micro patterns methods were used in [16]. When compared to gradient-based methods, these approaches provide less accuracy, particularly when the main purpose is to follow the flow lines [12]. The gradient phase angle presents the direction of the maximum intensity change. Therefore, the direction $\phi_{c}$ of a hypothetical edge that crosses the region centred on pixel 
$\left(i_{c}, j_{c}\right)$ is orthogonal to the gradient phase angle on $\left(i_{c}, j_{c}\right)$. Although this method appears simple and efficient, it suffers from nonlinearity due to the computation of the gradient phase angle and a discontinuity around 90 degrees [11]. Hence, generally for gradientbased methods, a window is applied or the consideration of a pixel neighbourhood is suggested. In a method proposed by Kawagoe and Tojo [16], a $2 \times 2$ pixel neighbourhood was considered. In order to extract a rough directional estimate, a straight comparison against four edge templates was made. Then, after averaging over a large region, a more distinct estimate was obtained. Similar approaches were followed in [18] and [19], where the tangent direction was evaluated based on pixel alignments relative to the fixed number of reference directions. The result was then averaged over a local window, using the least squares minimization for noise control. In a following subsection, we concentrate on the oriented direction, which is a necessary definition for boundary tracing of the prostate.

The techniques used to compute the tangent directions are efficient and robust. However, they can become computationally expensive if the local windows are large (greater than 19 pixels) or the number of directions to be computed is very high [11]. A more efficient implementation can be obtained by pre-computing the directional image over a discrete grid, followed by estimating the direction $\phi_{c}$ using Lagrangian interpolation [9].

As previously explained, the orientation is logically and practically specified for a block of pixels. In averaged squared gradients approach, an image is split into a set of $W \times W$ non-overlapping blocks and a single local ridge orientation is defined for each block. In a prostate image, there is no difference between local opposite ridge 
orientations; for example, in a local neighbourhood, the ridges oriented at 90 degrees and the ridges oriented at 270 degrees cannot be differentiated from each other. The same is true for the other opposite directions.

The calculation of the orientation of each image block, using a small neighbourhood of a generic pixel [12], is described below. According to the Average Squared Gradient (ASG) method [12], if $[\chi, \gamma]$ is a generic pixel in the boundary of the prostate image, the local ridge orientation at $[\chi, \gamma]$ is the angle that the boundary ridges form with the horizontal axis. In order to compute the orientation, a small square neighbourhood of pixels centred at $[\chi, \gamma]$ is involved in the computation.

From an analytical point of view, the gradient vector at $[\chi, \gamma]$ for a grey scale image $I$ is defined as:

$$
\left[\begin{array}{l}
G_{\chi}(\chi, \gamma) \\
G_{\gamma}(\chi, \gamma)
\end{array}\right]=\operatorname{sign}\left(G_{\chi}\right) \nabla I(\chi, \gamma)
$$

Since "doubling the angle and squaring the length of a vector" is equal to "square a complex number", we have:

$$
G_{s, \chi}+j \cdot G_{s, \gamma}=\left(G_{\chi}+j \cdot G_{\gamma}\right)^{2}=\left(G_{\chi}^{2}-G_{\gamma}^{2}\right)+j \cdot\left(2 G_{\chi} G_{\gamma}\right)
$$

using the following terms:

$$
\begin{aligned}
G_{\chi \chi} & =\sum_{W} G_{\chi}^{2} \\
G_{\gamma \gamma} & =\sum_{W} G_{\gamma}^{2} \\
G_{\chi \gamma} & =\sum_{W} G_{\chi} G_{\gamma}
\end{aligned}
$$


and averaging equation (2.2) using window $W$, the average gradient direction $\omega$ for $-\frac{1}{2} \pi<\omega<\frac{1}{2} \pi$ is defined by:

$$
\omega=\frac{1}{2} \angle\left(G \chi \chi-G_{\gamma \gamma, 2 G} \chi \gamma\right)
$$

Then the average ridge-valley direction $\theta$, with $-\frac{1}{2} \pi<\theta<\frac{1}{2} \pi$, is perpendicular to $\omega$ :

$$
\theta=\omega+\frac{\pi}{2}
$$

where $\Delta(x, \gamma)$ is defined as:

$$
\left\{\begin{array} { c } 
{ \operatorname { t a n } ^ { - 1 } ( \chi / \gamma ) } \\
{ \operatorname { t a n } ^ { - 1 } ( \chi / \gamma ) + \pi } \\
{ \operatorname { t a n } ^ { - 1 } ( \chi / \gamma ) - \pi }
\end{array} \quad \left\{\begin{array}{c}
\chi \geq 0 \\
\chi<0 \wedge \gamma \geq 0 \\
\chi<0 \wedge \gamma<0
\end{array}\right.\right.
$$

\subsubsection{Shape Model Concepts}

In many medical image interpretation problems, there is a vital need for an automated system. This suggests the use of models, which can describe and label the expected structure of the image. In some cases, image interpretation is almost impossible without prior knowledge of the anatomy especially when dealing with complex and variable structures, or images that suffer from noise and incomplete evidence [22].

Potential difficulty caused by structural complexity, noise and missing data can be solved by acquiring enough prior knowledge of the problem, and applying model-based methods in image analysis. We would like to apply knowledge of the expected shapes of prostate contours model to restrict our automated system to acceptable interpretations.

In addition, variability in an image of a biological object within and between subjects must be dealt with. This leads to the idea of deformable models. These models 
maintain the essential characteristics of the class of objects they represent, but can deform to fit a range of examples. In order to obtain specific models of the variable objects, we need to know how objects vary.

Among different model-based methods, generative models can significantly complete and generate possible images of target objects. One example would be a face model capable of generating convincing images of any individual and tracking their gesture. Using such a model, image interpretation can be described as a matching problem [22].

Providing statistical models for the shape and appearance of the desired objects in the given images, through learning their variation from a suitably annotated training set, is considered a powerful approach. We need a training set of images in which corresponding 'landmark' points have been marked on every single image. These data can be used to compute a statistical model of shape. Also by considering suitable pixel based feature such as texture, intensity or colour of the pixels within the shape's interior an appearance model of the object can be extracted. With enough training examples, these models are able to synthesize any image of normal anatomy. By obtaining the parameters that optimize the match between a synthesized model, original image and target image, the algorithm can locate all the structures represented by the model.

The empirical shape model used by this algorithm is described in subsection 2.3.4. This model is based on the four user input points, defining 4 quadrants of the shape which is incorporated with the pre-knowledge of the curve change in each of the quadrants. 


\subsection{Algorithm Description}

\subsubsection{Overview}

A general flowchart of our algorithm is presented in Figure 2.7. Note that several steps needs to be used only once during the entire tracing procedure (modules 1,2 and 3), while the remaining steps are being used as many times as the tracer runs around the prostate boundary (modules 4,5,6,7 and 8).The following subsections explains each module in detail.

\subsubsection{Image Pre-Processing}

Image pre-processing consists of three important steps: 1) image enhancement, which increases the contrast of the original dark image, where the objects are hardly recognizable; 2) statistical normalization, which provides a low variation in the greylevel values for the whole image; and 3) orientation image calculation, which helps the tracing algorithm to follow the prostate boundary. 


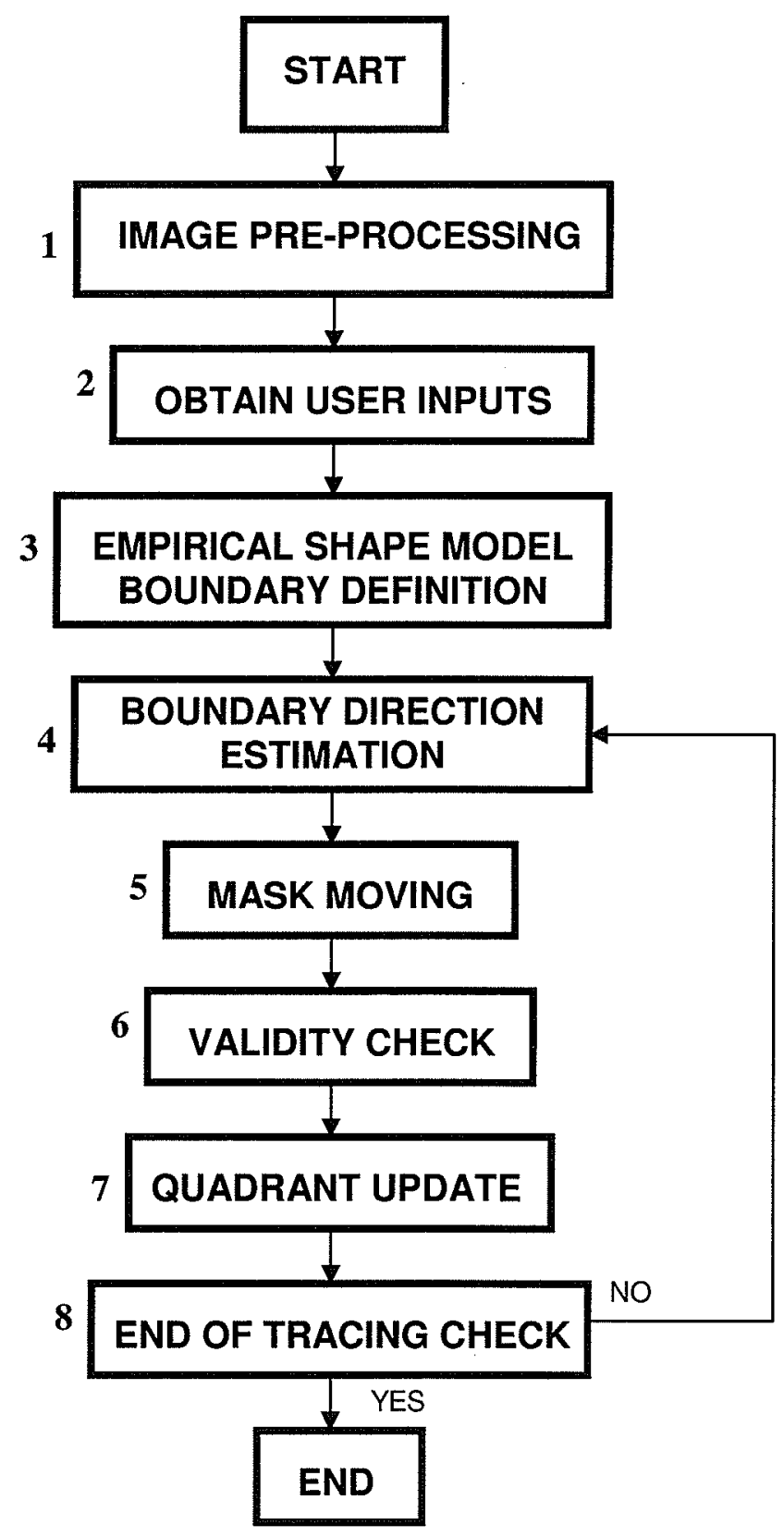

Fig. 2.7. General flowchart of the algorithm 


\subsubsection{Image Enhancement}

In the images involved in this work, we want to distinguish the prostate boundary. Slice 10 of the original inflated set is shown in Figure 2.8; it is difficult to distinguish where the prostate boundary is located. According to the original histogram (Figure 2.8), the number of high intensity pixels is quite few compared to the number of low intensity pixels. In order to achieve a better general view of the image, from which the initial points will be selected, we propose two modifications. First, the inversion of the original image should be found. Then the quality of the image should be modified using contrast stretching which is often called normalization.

The main purpose of contrast stretching is to change the range of pixel intensity values. This is the general equation of contrast stretching:

$$
P_{\text {out }}=\left(P_{\text {in }}-c\right)\left(\frac{b-a}{d-c}\right)+a
$$

Before the stretching can be performed, it is necessary to specify the upper and lower pixel value limits over which the image is to be normalized. Here we suggested 0 as the lower limit $a$ and 255 as the upper limit $b$. In addition, the highest and the lowest pixel values currently present in the image are 65536 as $d$ and 0 as $c$ respectively. Consequently, each pixel $P$ is scaled using the following function:

$$
P_{\text {out }}=\left(P_{\text {in }}\right)\left(\frac{255}{65536}\right)
$$




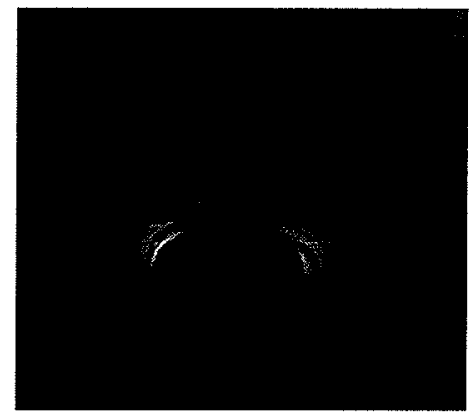

(a1)

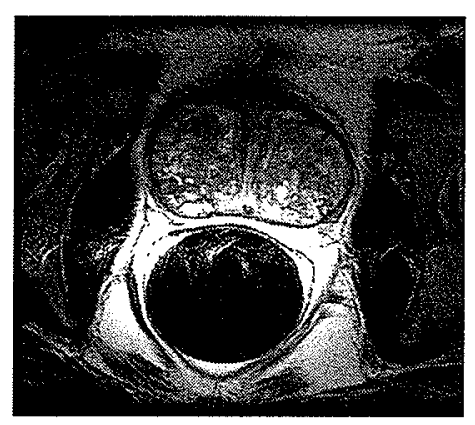

(c1)

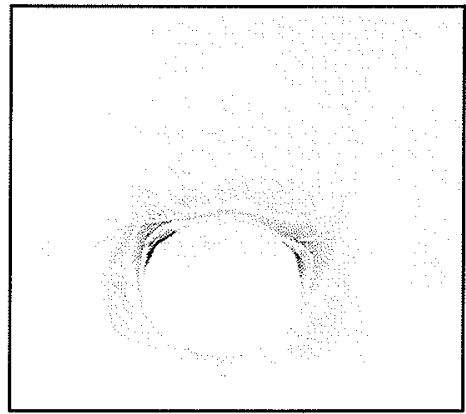

(b1)

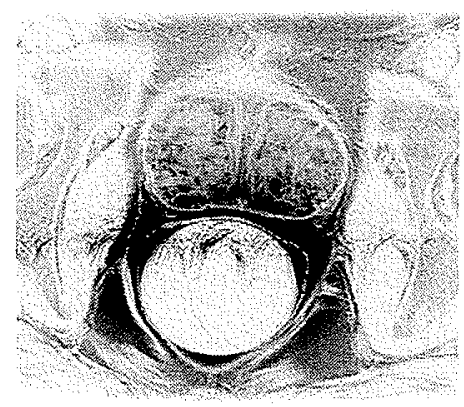

(d1)

Fig. 2.8. Histograms of the enhancing process: (a1) original image histogram; (b1) inverted image histogram; (c1) normalized histogram of non-inverted image; and (d1) normalized histogram of inverted image.

The images and histograms illustrating the original, intermediate and final steps are displayed in Figures 2.8 and 2.9. Note that after inversion, the original ridges become image valley structures, which are being followed by the tracing. As shown in Figure 2.8, the normalized inverted image gives the improved visualization. 


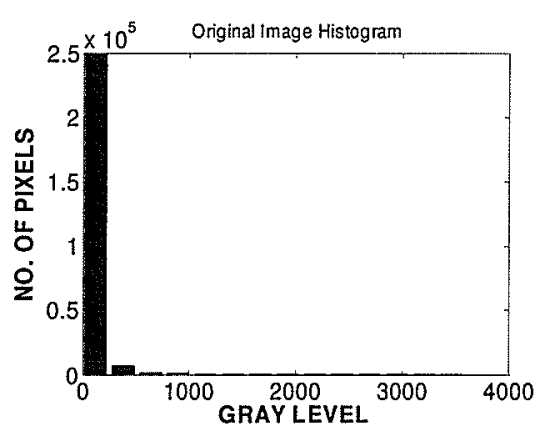

(a1)

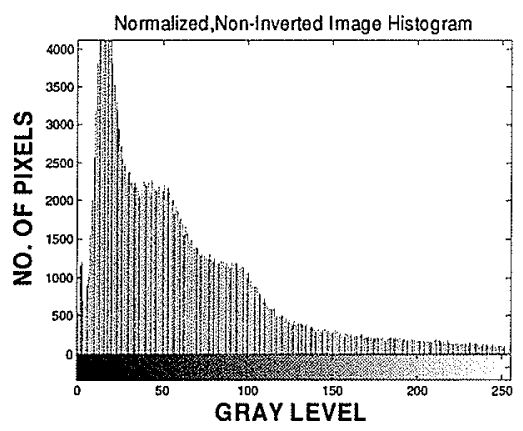

(c1)

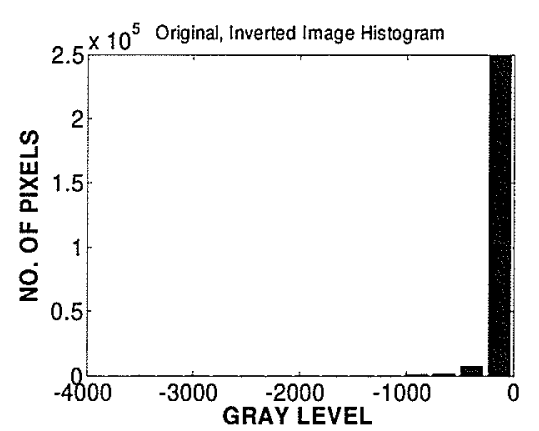

(b1)

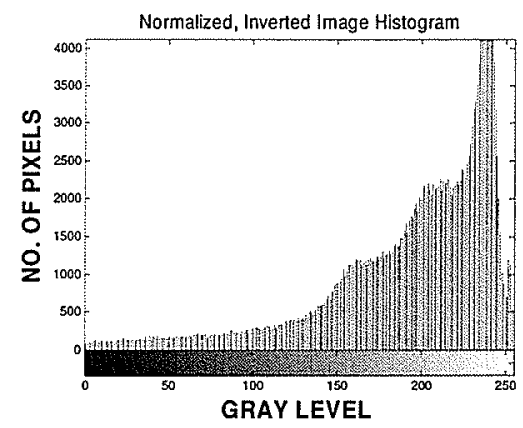

(d1)

Fig. 2.9. Images of MR prostate enhancing process: (a1) original image; (b1) inverted image; (c1) normalized non-inverted image; and (d1) normalized inverted image.

\subsubsection{Statistical Normalization}

A grey-level prostate image $I$ is defined as a $N \times N$ matrix, where $I_{j, k}$ represents the intensity of the pixel at the ith row and $j$ th column. The mean and variance of $I$ are defined as [21]:

$$
M(I)=\frac{1}{N^{2}} \sum_{i=0}^{N-1 N-1} \sum_{j=0} I(i, j)
$$

and

$$
\operatorname{VAR}(I)=\frac{1}{N^{2}} \sum_{i=0}^{N-1} \sum_{j=0}^{N-1}(I(i, j)-M(I))^{2}
$$

respectively. 
$M$ and VAR denote the estimated mean and variance of $I$ respectively; and $G(i, j)$ denotes the normalized gray-level value at pixel $(i, j)$. The normalized image is then defined as [21]:

$$
G(\chi, \gamma)=M_{0} \mp \sqrt{\frac{(I(\chi, \gamma)-M)^{2} V A R_{0}}{V A R}},
$$

where $M_{0}$ and $V A R_{0}$ are the desired mean and variance values, which are 0 and 1 respectively. Therefore, the above formula can be simplified to:

$$
G(\chi, \gamma)=\mp \sqrt{\frac{(I(\chi, \gamma)-M)^{2}}{V A R}},
$$

The main purpose of normalization is to reduce variations in the gray-level values along the ridges and valleys, which facilitates the subsequent processing steps. Normalization is a pixel-wise operation; therefore, it does not change the clarity of the ridge and valley structures. 


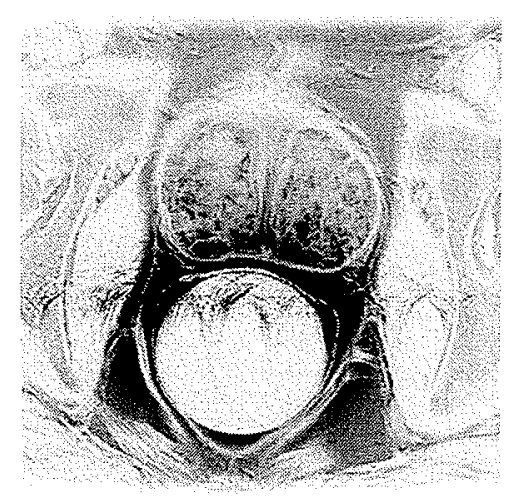

(a1)

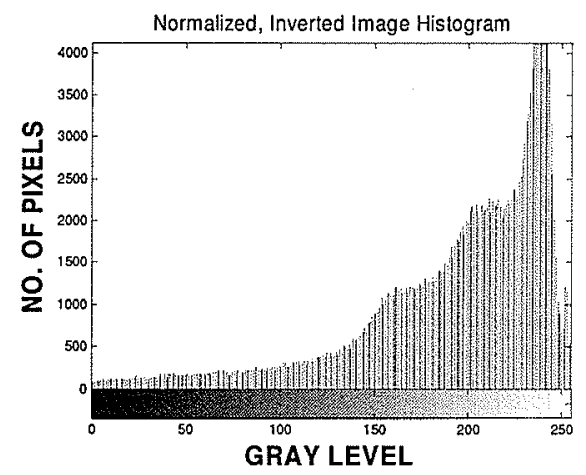

(b1)

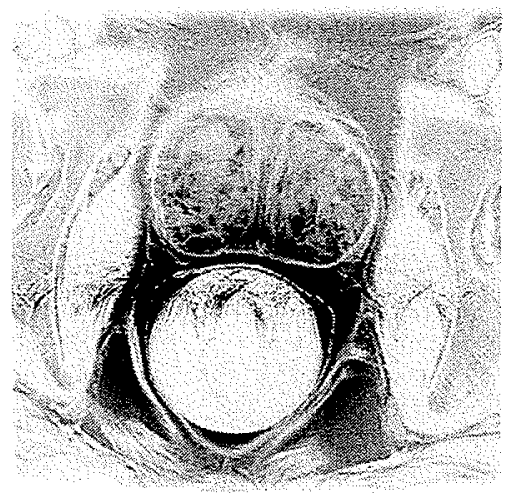

(a2)

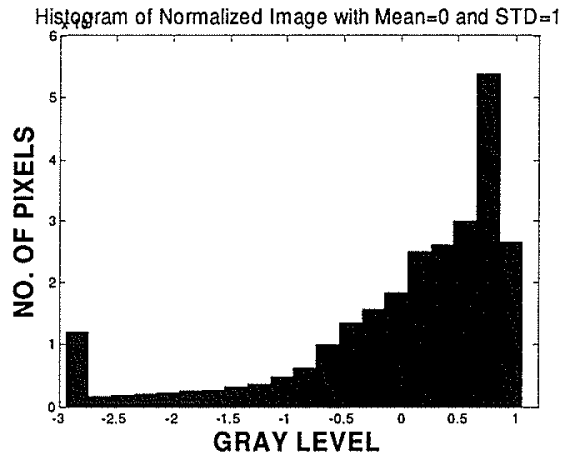

(b2)

Fig. 2.10. Images and histograms for: (a1) (b1) enhanced; (a2) (b2) general statistical normalization.

\subsubsection{Orientation Image Calculation}

To determine the orientation estimation, we used the "Average Squared Gradient Method" [12]. A general block diagram of this module is presented in figure 2.11. Due to the presence of noise and the corrupted ridge and valley structures in the input image and slowly variation of the local ridge orientation in a local neighbourhood, low-pass filters in the form of Gaussian filters are used three times, in the whole process of computation. These Gaussian filters can not be combined together, due to the non-linear operators which are involved in between. The image initially is divided into $7 \times 7$ non-overlap 
windows, as the same size of the first group of the Gaussian filters. The size of the window is dependent on the size of the structure to be extracted (prostate boundary) and the image resolution. This size is practically selected by trial and error criterion.

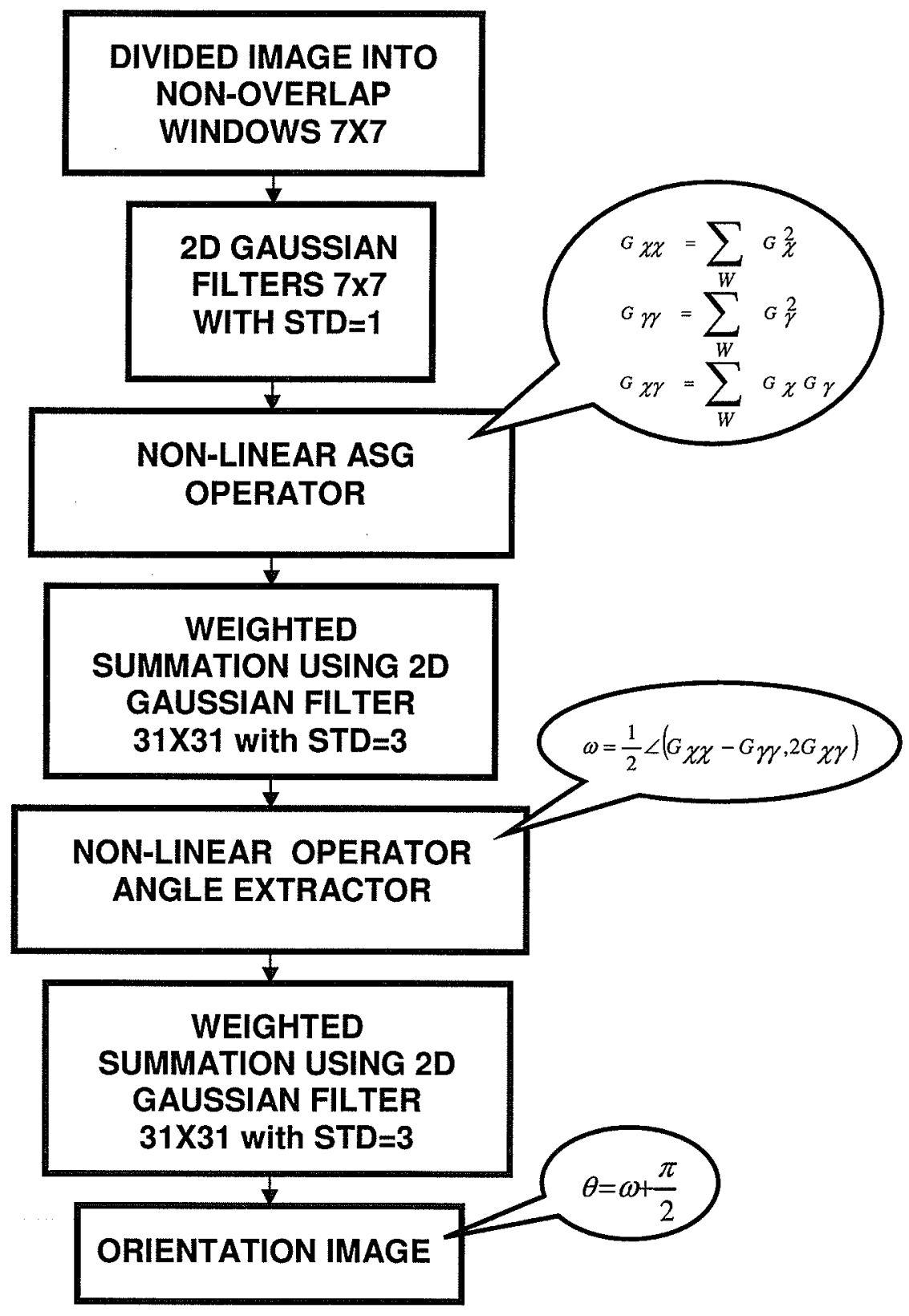

Fig. 2.11. General block diagram of the orientation image calculation. 
Given a normalized image $G$, the main steps of the algorithm are as follows:

1) Generate a $7 \times 7$ Gaussian filter, with a standard deviation of one. Compute the gradient of the Gaussian filter in the $x$ and $y$ directions.

2) Compute the gradients $\partial_{\chi}(i, j)$ and $\partial_{\gamma}(i, j)$ at each $(i, j)$ using the above Gaussian filters. Computation of the orientation is being performed over the $7 \times 7$ block.

3) Obtain the covariance data for the image gradients, which are $\partial_{\chi, \chi}(i, j)$, $\partial_{\gamma, y}(i, j)$ and $\partial_{\chi, \gamma}(i, j)$ at each pixel $(i, j)$.

4) Using a $31 \times 31$ Gaussian core with a standard deviation of five, the above results are smoothed, i.e. a weighted summation of the data.

5) Estimate the local orientation of each block $7 \times 7$ centred at pixel $(i, j)$ using the following equation:

$$
\omega_{i, j}=\frac{1}{2} \tan ^{-1}\left(\frac{G_{\chi, \chi}-G_{\gamma, \gamma}}{2 G \chi, \gamma}\right)
$$

6) Smoothing is again performed using the same Gaussian core as step 4.

7) The angle $\theta$ is extracted (with a 90-degrees difference) when the orientation image is obtained.

Computing the local ridge orientation at $(i, j)$ using this algorithm, a smooth orientation field estimate is obtained. In Figure 2.12, the effect of removing both of the filters in steps 4 and 6, applied after nonlinear operators and their effect size on the clarity 
of the orientation image are verified. According to the results, no improvement will be achieved after increasing the filter size to $31 \times 31$ with $\mathrm{STD}=5$.

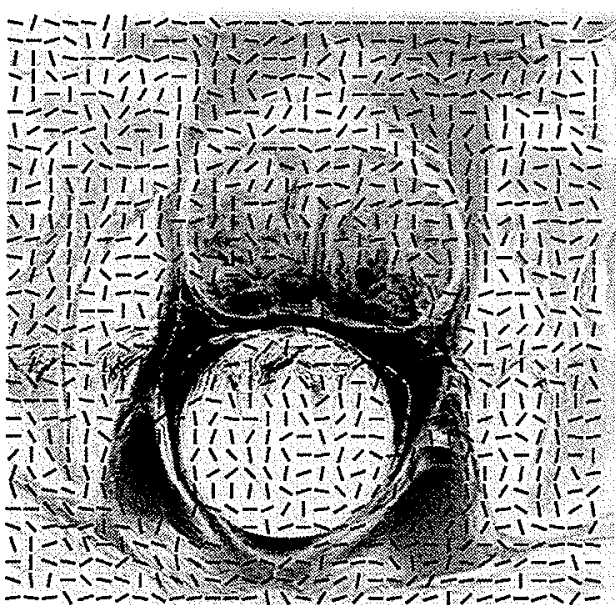

(a)

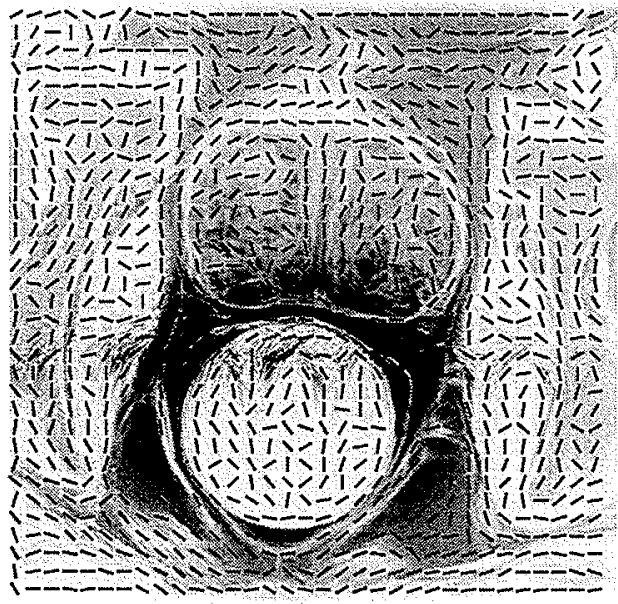

(c)

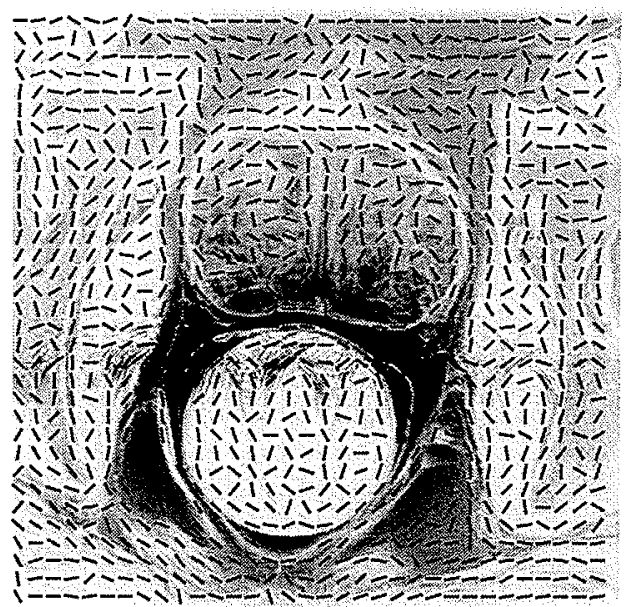

(b)

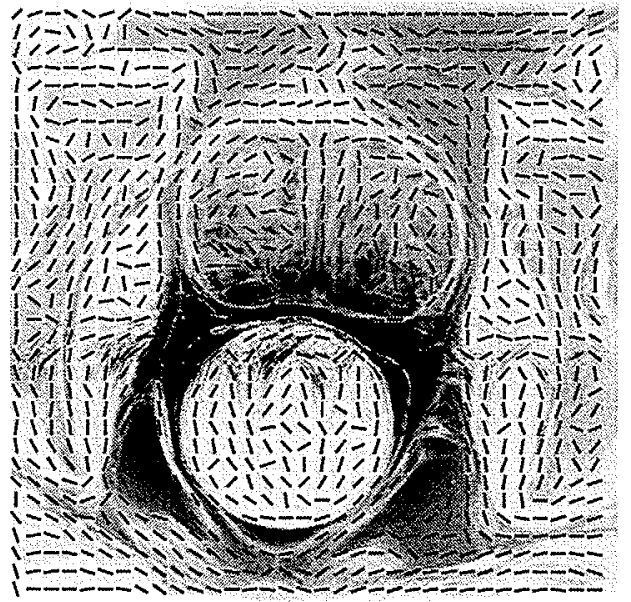

(d)

Fig. 2.12. Images showing the effects of the smoothing filters after non-linear operators and their size effect: (a) no smoothing filter; (b) filter size 19 and STD=3; (c) filter size 25 and $S T D=4$; (d) filter size 31 and $S T D=5$.

Figure 2.13 shows an example of the output of the gradient operator (linear), covariance matrixes (nonlinear operators as AGS components) and angle extractor (nonlinear). Note that mathematically, this angle represents the direction that is orthogonal to the domain direction of the Fourier spectrum of the $W \times W$ window [21]. 
Note that the smoothing operation is performed at the block level.

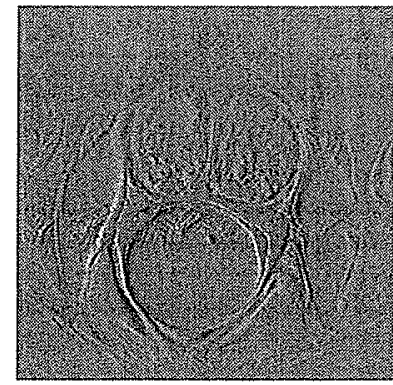

(a1)

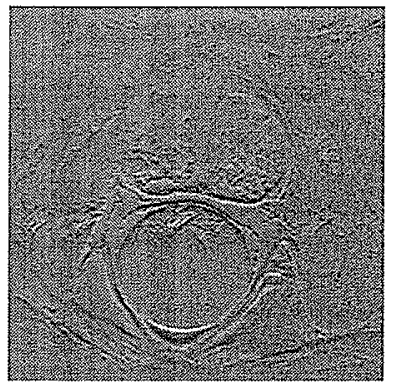

(a2)

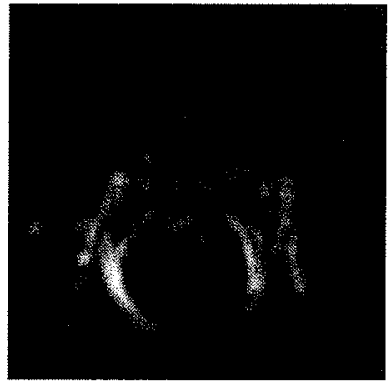

(b1)

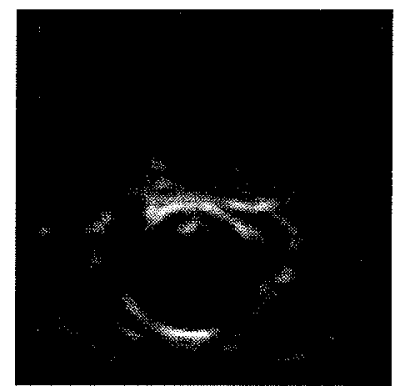

(b2)

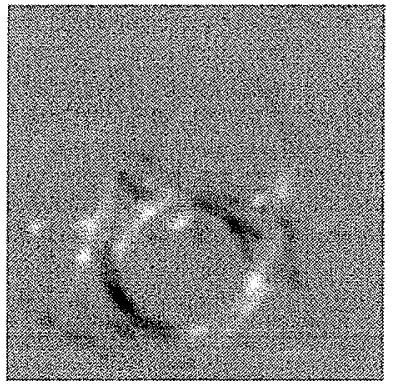

(b3)

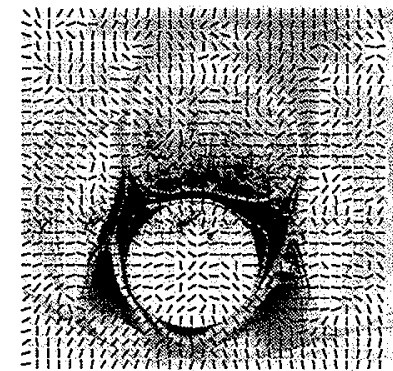

(c1)

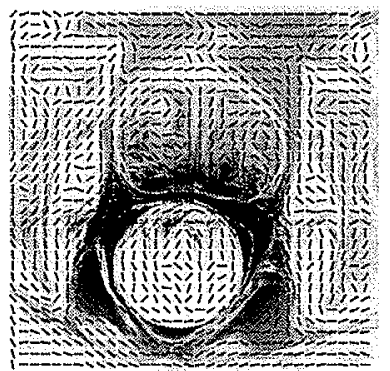

(c2)

Fig. 2.13. Images showing outputs of different steps of orientation image computation, assuming $G$ are the image and block levels of size $7 \times 7:$ (a1) $G_{\mathrm{X}}$; (a2) $G_{\mathrm{Y}}$; (b1) $G_{\mathrm{XX}}$; (b2) $G_{\mathrm{YY}}$; (b3) $G_{\mathrm{XY}}$; (c1) gradient directions in block level; and (c2) directional field in block level. 


\subsubsection{User Inputs}

Our proposed shape model is an estimation of the valid search area around the object of interest and a prediction of the valid curve change in specific areas of the object boundary. As previously mentioned, the user needs to select four pixels in the neighbourhood of the prostate boundary on each slice; a rectangular area is then constructed based on these four points, which covers the entire prostate as tightly as possible.

As long as the four points are located in the approximate middle of the prostate boundaries on each side, which represent the furthest extreme of the prostate, the resulting empirical shape model is reliable. The main reason this characteristic is defined for our shape model is to prevent the tracing algorithm from being distracted by the other neighbouring organs, e.g., the bladder (superior), the rectum (posterior), and the leg bones (left and right). These bound restrictions prevent the tracer from moving beyond these limits. Figure 2.14 depicts a sample boundary for slice 9 of the inflated data set.

The other aspect of our empirical shape model is explained in the following subsection.

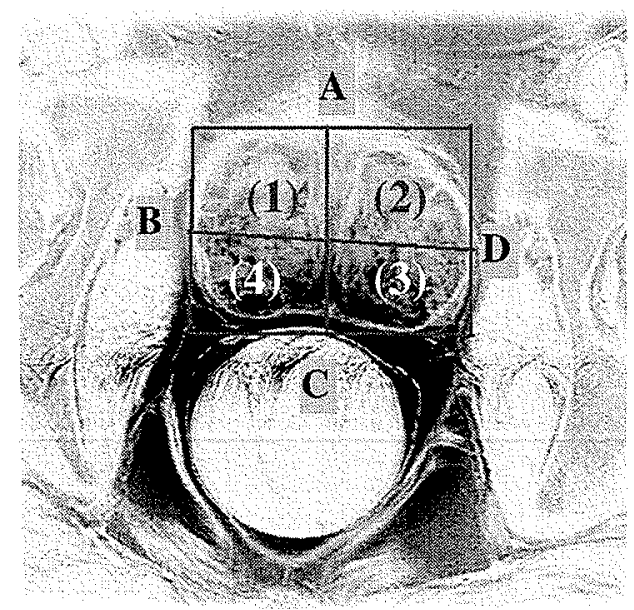

Fig. 2.14. Estimation of the valid boundary area, which uses defined points shown on $\mathrm{A}, \mathrm{B}, \mathrm{C}, \mathrm{D}$ and quadrants $1,2,3,4$. 


\subsubsection{Empirical Shape Model Definition}

The empirical shape model is presented in Figure 2.15. The worst case where all the organs surrounded the prostate and the high chance of the tracer being distracted by the neighbouring organs considered in this method. According to this shape, the valid direction of the prostate boundary curve in each quadrant (defined using the four initial input points) is mapped in a corresponding look up table. The boundary direction estimation module uses this information.

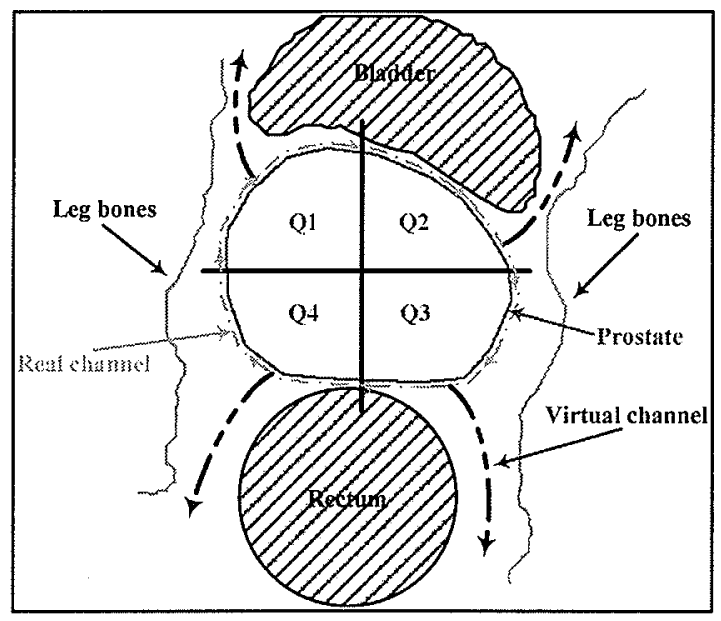

Fig. 2.15. Empirical shape model of prostate

\subsubsection{Boundary Direction Estimation}

Recall the fingerprint classification research developed by Maio, which applied a direct ridgeline tracing method [9]. At each step, the algorithm computed a new point $\left(i_{t}, j_{t}\right)$ by moving $\mu$ pixels from the current point $\left(i_{c}, j_{c}\right)$ along the direction $\phi_{c}$; this represents the ridgeline local direction and can be computed as the tangent to the ridge at $\left(i_{c}, j_{c}\right)$, by taking the closest local maximum in a specified area along the previous direction. 
We have modified Maio's approach. Instead of relying on the definitions of local maxima and ridges in each step, which provides a very fine scale for the ridgeline surrounding the prostate, the orientation image is computed only once at the beginning. Then, a $3 \times 3$ (selected based on trial and error) moving window is used, which finds the average $D F$ corresponding pixels; from these, the direction of the tracing curve can be estimated. Figure 2.16.c illustrates the above concept; note that this orientation is not directed.

Once the direction is estimated, the results need to be interpreted in order to estimate the corresponding orientation. Using the coordinates of the four initial inputs, the prostate's centre of mass is estimated. According to the location of the four points and the centre of mass, the four quadrants of the prostate are defined (Figure 2.16.a). A look up table is defined, which presents the valid and invalid directions in each quadrant and the appropriate maps of the movement pattern at the pixel level (Figure 2.16.d). This look up table has been estimated according to the general shape of the prostate and considering the effects of the other organs surrounding it. Using this look up table, the tracer can follow the ridgeline quite closely. The results are located in $\left[-90^{\circ},+90^{\circ}\right]$. Altogether, thirteen different directions with equal steps have been defined. Figure 2.16.d illustrates how a unique direction can be interpreted from different patterns of pixel movements, according to its spatial location. 


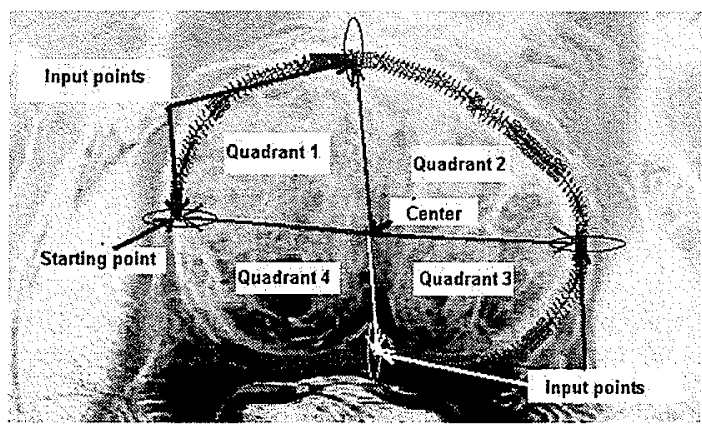

(a)

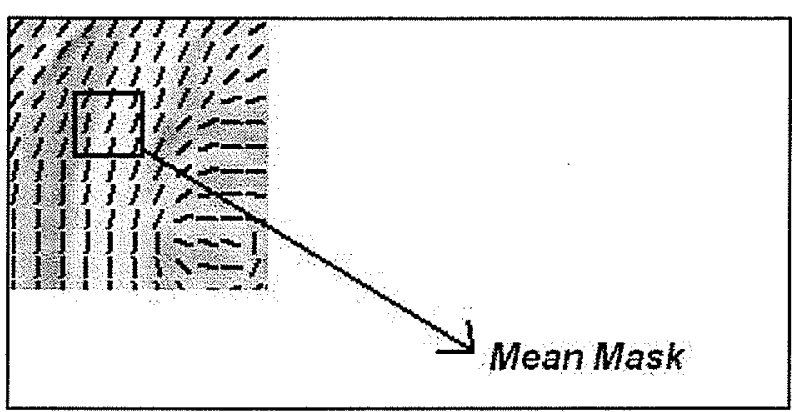

(b)

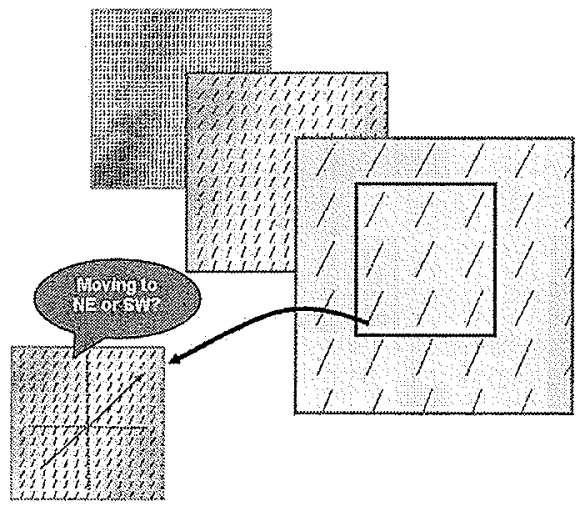

(c)

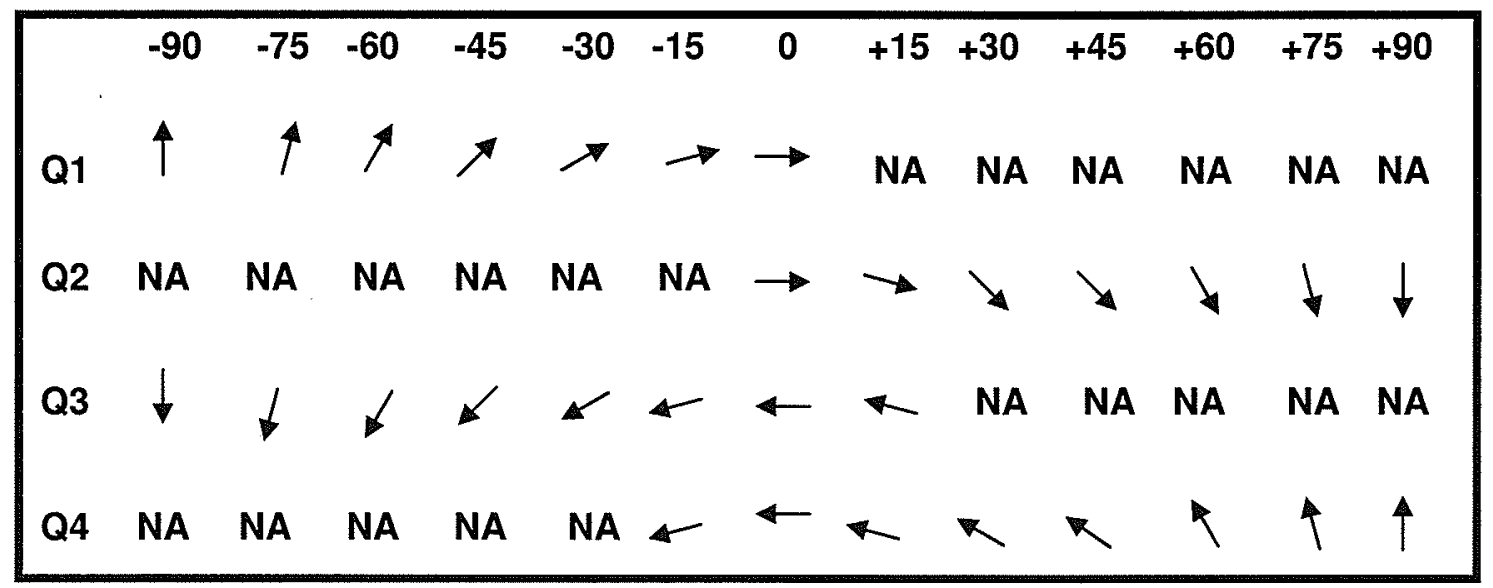

(d)

Fig. 2.16. Estimation of the boundary direction: (a) definition of quadrants, initial points and centre of mass; (b) the average of the pixel field direction covered by the mask is used for calculating the new position; (c) emphasising the concept of orientation without direction; and (d) a complete look up table showing the orientation patterns based on the quadrant location. 


\subsubsection{Mask Movement}

According to the output of the previous module, that estimates the curve direction of the prostate boundary in the spot, the window mask moves to a new location. The map of the next direction is defined in the form of the number of pixels in the direction of $x$ axis and $y$-axis.

\subsubsection{Quadrant Update}

As the tracer runs along the boundary of prostate, its current location in the form of quadrant information needs to be updated. This is important for the boundary direction estimation module in order to refer to the relevant look up table.

\subsubsection{End-of-Tracing-Change}

The algorithm runs until the stop criterion is true. At each step, it updates the quadrant label looking for the fourth quadrant. When the tracer is close to the starting point (about some pixels) selected in quadrant one, the entire boundary has been traced (Figure 2.17).

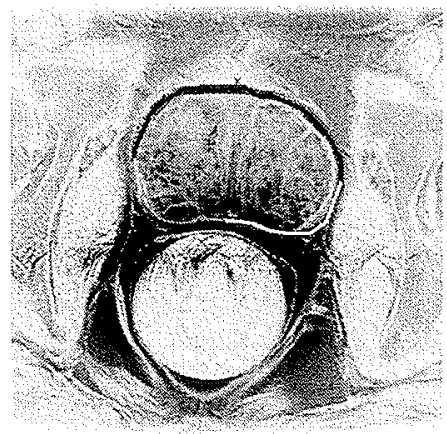

(a)

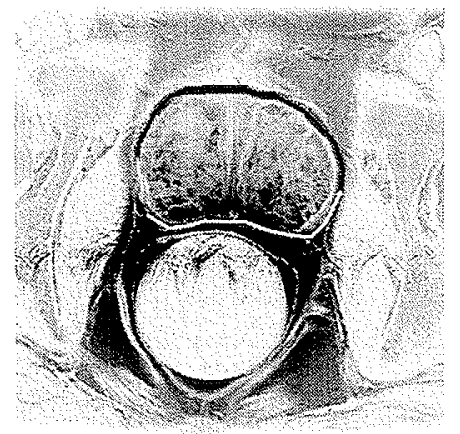

(b)

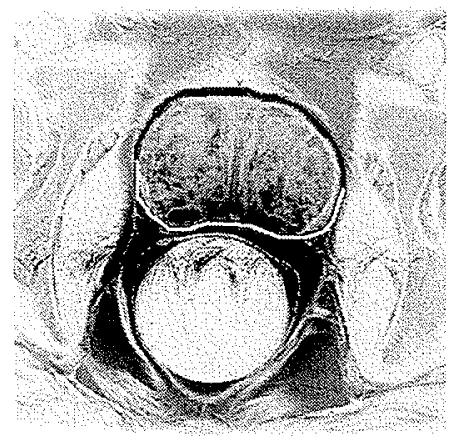

(c)

Fig.2.17. The completion of the tracing procedure: the algorithm continues to trace the boundary until the tracer moves into the neighbourhood of the starting point in quadrant four: (a) end of tracing in quadrant 3; (b) tracing in quadrant 4; (c) end of tracing in quadrant 4 or end of the tracing procedure. 


\subsection{Performance Evaluation}

Using MRI as the selected imaging modality provides essential information. This information allows us to obtain quantitative pathologic and clinical evaluations [29].

Accurate segmentation in medical imaging is important, due to its direct impact on disease detection, target definition for treatment and monitoring of disease progression for response [29]. High performance evaluation of the segmentation can be achieved by two key concepts: reproducibility, or statistical validation and accuracy.

In order to conduct the performance evaluation of the image segmentation, a 'Gold Standard' is required. This Gold Standard is defined using a simple binary truth, which indicates the true (target) tissue class per voxel. Manual segmentation of the prostate on the both deflated and inflated data set was provided by an expert radiologist (explained earlier in details in subsection (2.1)) which is considered as the Gold Standard.

\subsubsection{Dice Similarity Coefficient}

To measure accuracy, one of the simplest spatial overlap metrics is the Dice Similarity Coefficient (DSC) [30]. It is both a spatial overlap index and a reproducibility validation metric, frequently use in observer performance studies [35]. Flesis termed it the "proportion of specific agreement" [31]. The DSC value ranges from zero (no spatial overlap between two sets of binary segmentation results) to one (complete overlap). It has been adapted to validate the segmentation of the peripheral zone of the prostate gland in prostate brachytherapy in [35]. The DSC is used to assess the accuracy of the segmented prostate in this work. Other metrics for statistical validation of accuracy include the Jaccard similarity coefficient [32], odds ratio [33], receiver operating characteristic 
analysis [33], [34], mutual information [34], [35] and distance-based statistics [36], [37]. The validation test method used in this work is briefly explained in the following paragraph.

The DSC measures the spatial overlap between two segmentations $A$ and $B$. It is defined as $D S C(A, B)=2(A \cap B) /(A+B)$, where the numerator is the intersection between the two segmentations. It can also be defined as [53]:

$$
D S C=\frac{2 a}{2 a+b+c}
$$

where $a$ is the area correctly classified as the prostate, $b$ is the area incorrectly classified as the prostate, and $c$ is the area incorrectly classified as the background (Figure 2.18). 


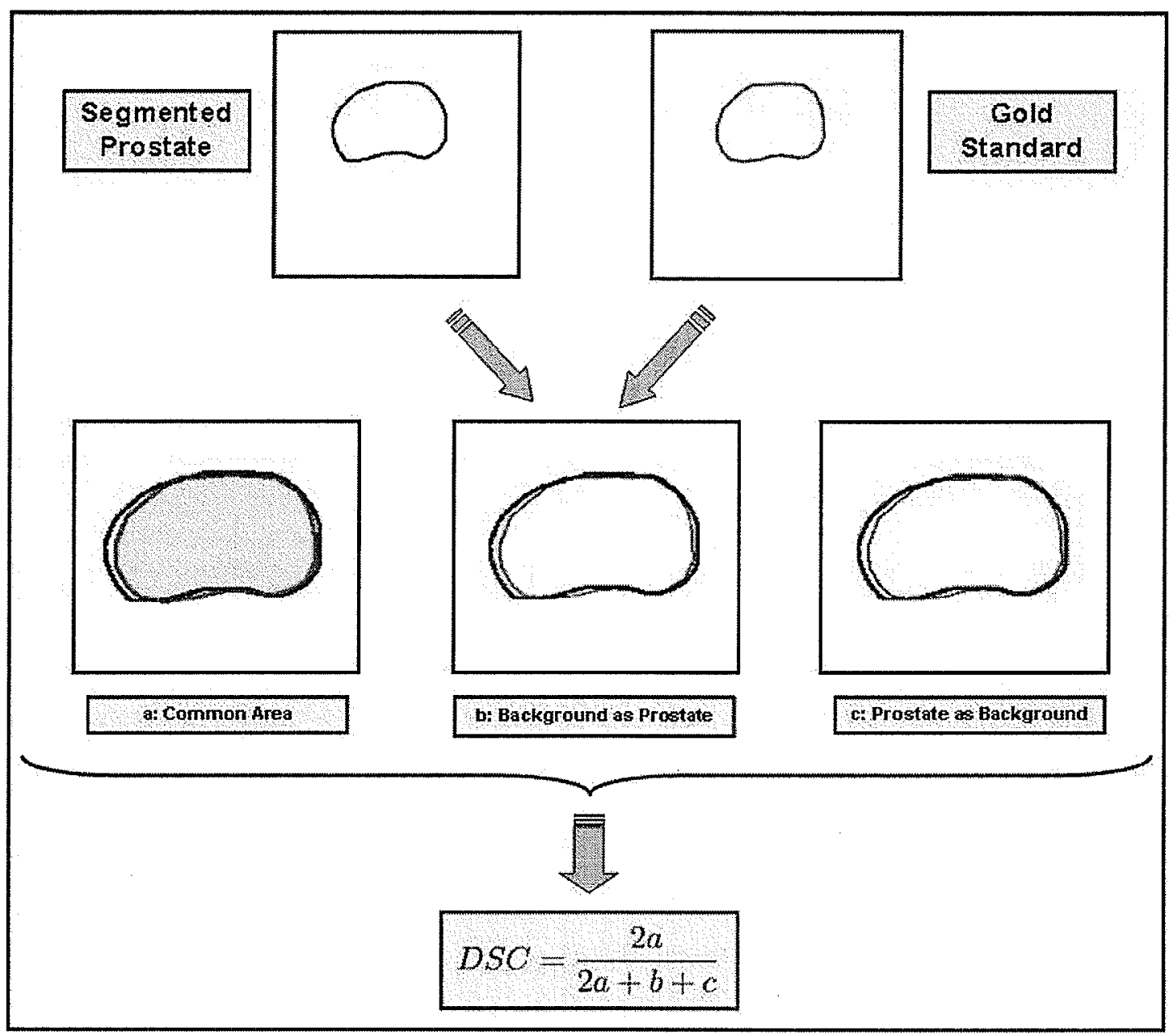

Fig. 2.18. DSC calculating procedure: The above flowchart indicates the procedure for calculating DSC using the Gold Standard and the result of suggested semiautomatic segmentation method.

Our goal was to validate segmented boundary of prostate in both of the inflated and deflated data sets in 10 trials. In each trial, for each single slice, the user selects four different input points. Therefore, we can verify how much the segmentation is depended on the selection of the initial points. Two different approaches were considered to evaluate the accuracy and reproducibility performance of our semi-automatic segmentation method. 


\subsubsection{Mean and Variance Analysis}

The first statistical method finds the mean of $D S C$ for each slice in a trial of $N$ samples, as follows:

$$
\overline{D S C_{i}}=\frac{1}{N} \sum_{i=1}^{N} D S C_{i},
$$

where $\overline{D S C_{i}}$ is the average $D S C$ over all $N$ trial corresponding each individual slice and $D S C_{i}$ is the spatial overlap corresponding each single slice of a trial. Then its uncertainty can be estimated by the standard deviation of the $D S C_{i}$ around $\overline{D S C_{i}}$ as:

$$
S T D_{D S C_{i}}=\sqrt{\frac{1}{N} \sum_{i=0}^{N}\left(D S C_{i}-\overline{D S C_{i}}\right)^{2}}
$$

In this work, the number of trials, $N$, is equal to 10 . The overall mean and the overall $S T D$ per data set, representing the intra-operative accuracy of the segmentation can be calculated as following:

$$
\begin{gathered}
\overline{D S C}_{\text {Overall }}=\frac{1}{19} \sum_{i=1}^{19} \overline{D S C}_{i}, \\
S T \overline{D S C}_{\text {overal }}=\sqrt{\frac{1}{19} \sum_{i=1}^{19}\left(\overline{D S C}_{\text {Overall }}-\overline{D S C}_{i}\right)^{2}} .
\end{gathered}
$$




\subsubsection{Statistical Ranking Analysis}

A box plot is useful because a number of robust estimates of the median and data spread can be shown in a single plot. This concept is based on rank statistics. Compared to mean and variance analysis, it is more robust and less sensitive to extremely large or small values in the data set. We are applying both of the analysis (Mean and Variance, and Statistical Ranking) to compare the results of analysis together.

To explain the related concepts and definitions, consider the results of semiautomatic segmentation of the previously mentioned data set, consisting of 10 trials for each of our clinical data sets (each data set has 19 slices). A $2 D$ array of data is provided, with the rows representing the slice number and a box being plotted for each column. The positions of the box and the lines extending from it (the whiskers) are based on rank statistics. First, the values in the data set are ordered and then the median of $n$ samples is calculated; the line in the middle of the box represents the median ( $50^{\text {th }}$ percentile). The upper and lower edges of the box are placed at the $25^{\text {th }}$ and $75^{\text {th }}$ percentiles, respectively while the middle percentile or median lying in the middle of the box. In other words, the first and last $25^{\text {th }}$ percentiles lie above and below the box, respectively. Lastly, the whiskers are drawn at a distance from the median of 1.5 times the inter-quadratic range (difference between the $25^{\text {th }}$ and $75^{\text {th }}$ percentile), or to the object furthest away; this is dependent on which value occurs first. All objects falling outside the whiskers are drawn separately as outliers. Figure 2.17 presents a sample box plot.

Regarding accuracy, for $D S C=1$ there is perfect overlap and for $D S C=0$ there is no overlap between the 'Gold Standard' and semi-automatically segmented regions. 


\section{CHAPTER 3}

\section{Results and Discussions}

The various aspects of the developed segmentation technique are evaluated in this chapter. A qualitative comparison between the semi-automatic approach and the manual segmentation is provided. Results from single trial segmentation are presented, as the obtained boundaries along with their corresponding manual segmentations projected back on the enhanced images; refer to Figures 3.2 and 3.3 for illustration. Subsequently, we consider the method's accuracy and robustness (qualitative comparison) with respect to the four manually different selected initial inputs required to perform the segmentation as they meet the algorithm requirements for the input points in ten trials. A single user performs the segmentation algorithm 10 times for each of the slices in each data set. The outcome of this approach is compared with the corresponding Gold Standard on a slice-by-slice basis in 10 trials using DSC.

In order to calculate the DSC value, three quantities of interest are specified. In the subsection (2.4.1), we referred to these variables $\boldsymbol{a}, \boldsymbol{b}$ and $\boldsymbol{c}$, corresponding to the area of intersection, the area of prostate segmented as background, and the area of background segmented as prostate; refer to Figure 3.1 for illustration. This figure shows each of the above three areas in a binary image, which aids the auxiliary algorithm to calculate these three variables according to corresponding number of pixels. 
The mean and variance of the DSC are calculated, per slice for each trial of 10 mentioned before. In order to display the results, two graphs showing the per slice DSC mean and corresponding DSC variance are considered (Figures 3.4 and 3.5). Furthermore, the per slice median of the DSC, along with the graphical display of the DSC values located around the median values, are depicted using box plots in Figure 3.6.

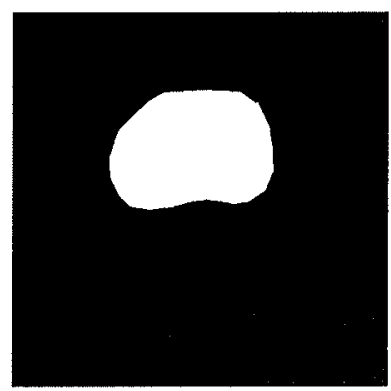

(a)

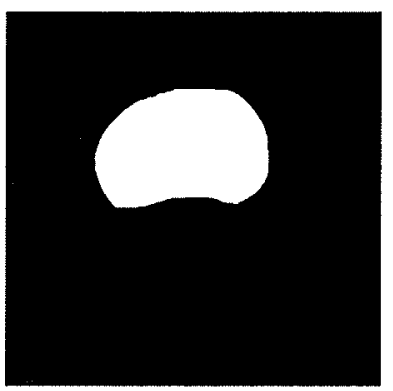

(b)

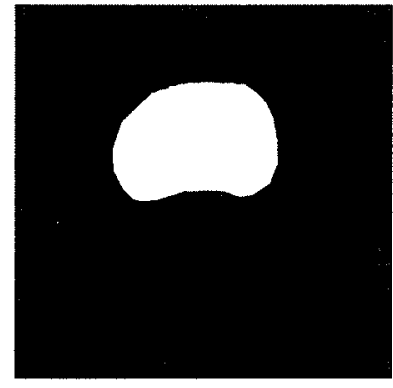

(c)

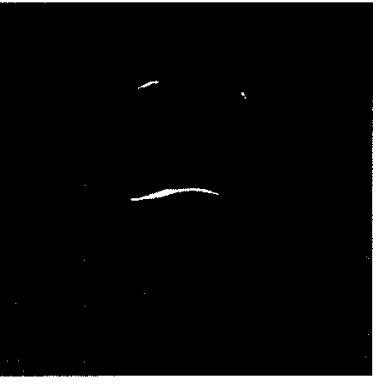

(d)

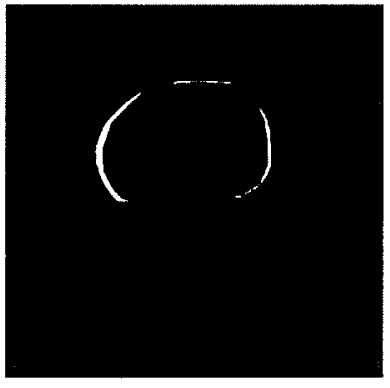

(e)

Fig. 3.1. Pictorial show of DCS components for performance evaluation: (a) filled contour of Gold Standard; (b) filled contour of segmented prostate; (c) area of intersection; (d) area of prostate segmented as background; and (e) area of background segmented as prostate. Images correspond to the inflated set, slice number 10. 


\subsection{Qualitative Comparisons}

Typical results are displayed for the inflated and deflated data set in Figures 3.2 and 3.3 respectively. The prostate boundaries segmented by our semi-automatic method are depicted in blue and the corresponding results obtained manually by the radiation oncologist are depicted in red.

The first group of pictures correspond to the inflated data set (Figure 3.2). The results show that the slices located in the middle of the data set (slices 6 through 16) comply very closely with the manual results. For the most inferior and superior slices of prostate, the algorithm had difficulty following the boundary wall. In the inferior slices, due to image contrast artifacts introduced by the endorectal coil the right and left side boundaries are confusing (note that the inferior slices of prostate correspond to the inferior end of the coil where there is a very little inflation compared with middle slice); in the superior slices, the prostate was located very close to the bladder, resulting in a confused boundary to be segmented. These results also show that the algorithm generally overestimates the boundary for most of the slices, however, the amount of the overestimation is not consistent where the search area is not tight enough.

A similar outcome is true for the second data set, where a deflated endorectal coil was used (Figure 3.3). However, the number of the slices for which the segmentation substantially deviates from the Gold Standard is greater than for the first group. Similar to the first group, the non-consistent overestimated boundary is one of the obvious results. Overall, the pictorial results show our algorithm works with better accuracy for the inflated data set. 

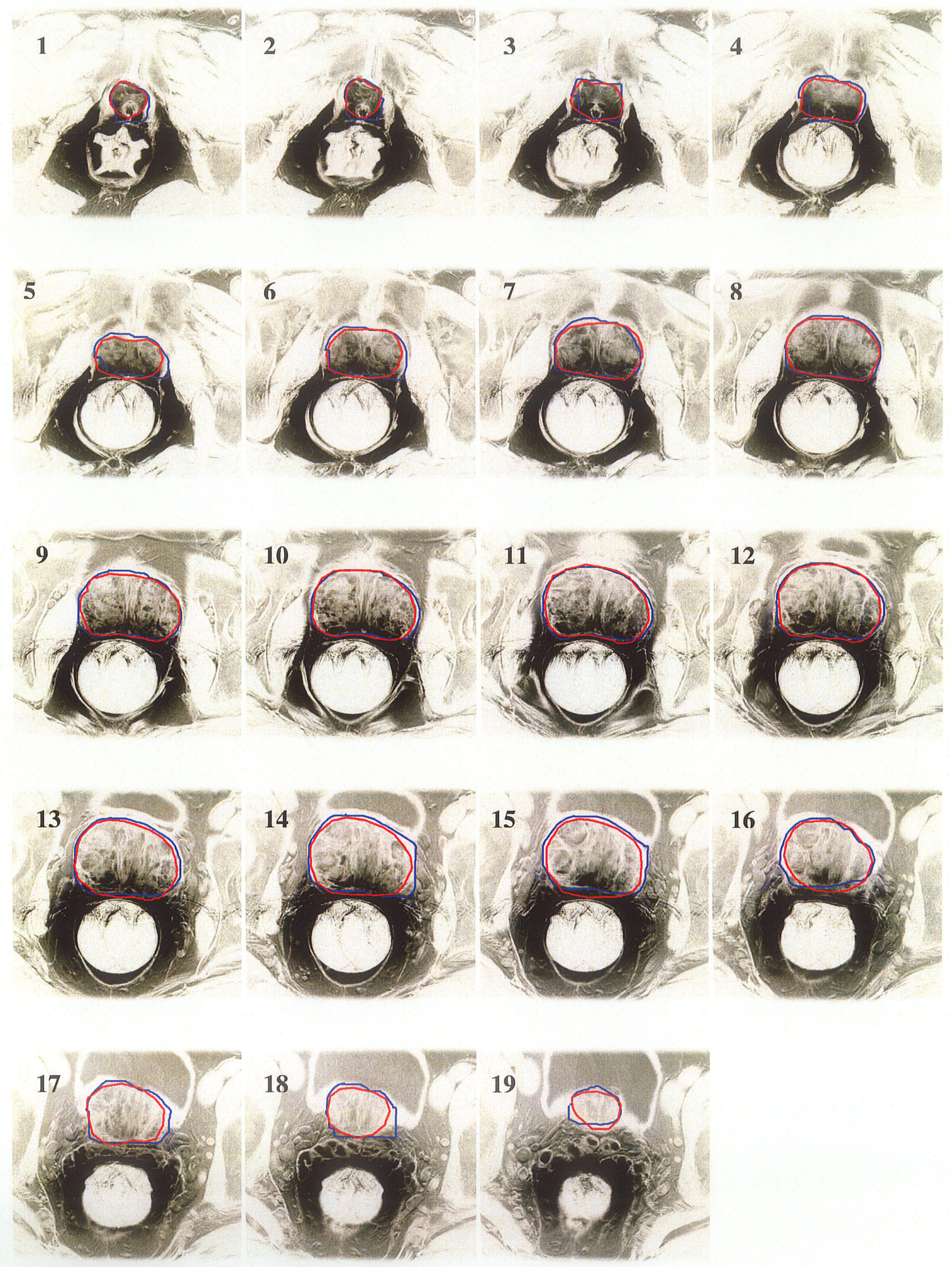

Fig. 3.2. Comparisons of manual (red) and semi-automatic (blue) segmentation. Images correspond to the inflated endorectal coil case. 

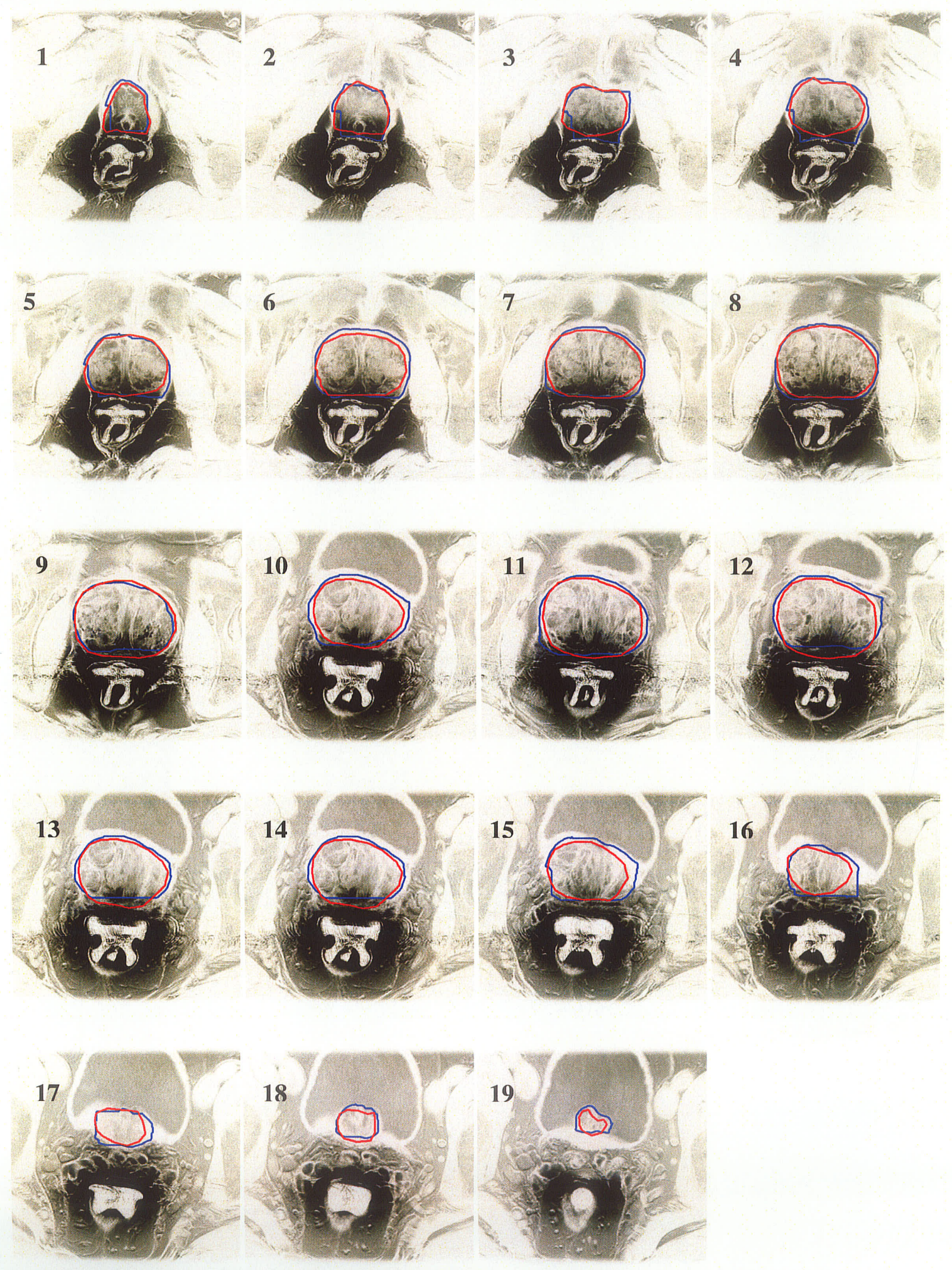

Fig. 3.3. Comparisons of manual (red) and semi-automatic (blue) segmentation. Images correspond to the deflated endorectal coil case. 


\subsection{Quantitative Comparisons}

As previously discussed in section (2.4), two different techniques were used to quantitatively estimate the accuracy and reproducibility of the proposed method; in the first approach, the mean and variance of DSC were extracted for each of the ten-segmented prostate, on each individual slice. These results are displayed in two separate graphs, corresponding each data set (Figures 3.4 and 3.5). Recalling subsection (2.4.2), the mean and the variance of individual slices are calculated using formula (2.17) and (2.18).

The overall mean DSC values are 0.90 (ranged 0.834-0.952 in figure 3.5) for the inflated set and 0.88 (ranged $0.700-0.965$ in figure 3.4 ) for the deflated set. The corresponding overall variance values are 0.001 for the inflated set and 0.004 for the deflate set. These results show that the mean value of the DSC for the inflated set is higher than for the deflated set and its variance is lower. Considering this statistical analysis, the introduced segmentation method is more accurate and reliable for the inflated set. Also referring to the DSC variance results in the same figures, slice number 19 in the deflated set and slice numbers 1 in the inflated set show the most variations in DSC values, when they are subjected to the change of the initial points.

Box plots can give another graphical depiction of these results. Figure 3.6 shows this concept for each of the data sets. The resulted DSC medians in both of the graphs comply almost the same as the previous analysis. According to the graph for the inflated set, all of the median values are located between 0.84 and 0.95 while the other data set ranged between 0.73 to 0.97 .This shows that the results of the algorithm are more accurate for the inflated set. The same slices (slice number 19 in the deflated set and 1 in the inflated set) represent the worst DSC variance similar to the previous analysis. 
Referring to the box plot outcomes, the number of the slices with the DSC outside the whisker bounds in the inflated set is more than the deflated set (about 3 times); however, the size of the boxes is almost the same in both sets. It demonstrates that the method is highly dependable on the initial points in the deflated set especially for inferior and middle slices; however, for the superior slices, the resulting boundaries appear more random. This illustrates that the developed technique for the deflated set yielded more robustness concerning the initial points.

Comparing all these observations, our semi-automatic method worked better for the inflated set regarding accuracy and was more reproducible for the deflated set. Recalling section (2.1), the average DSC obtained manually by a single expert, was 0.91 for the inflated set and 0.92 for the deflated set; our algorithm approaches the above average DSC values. Therefore, the suggested algorithm can approach the uncertainty of the radiation oncologist ability to draw prostate boundary consistently. 

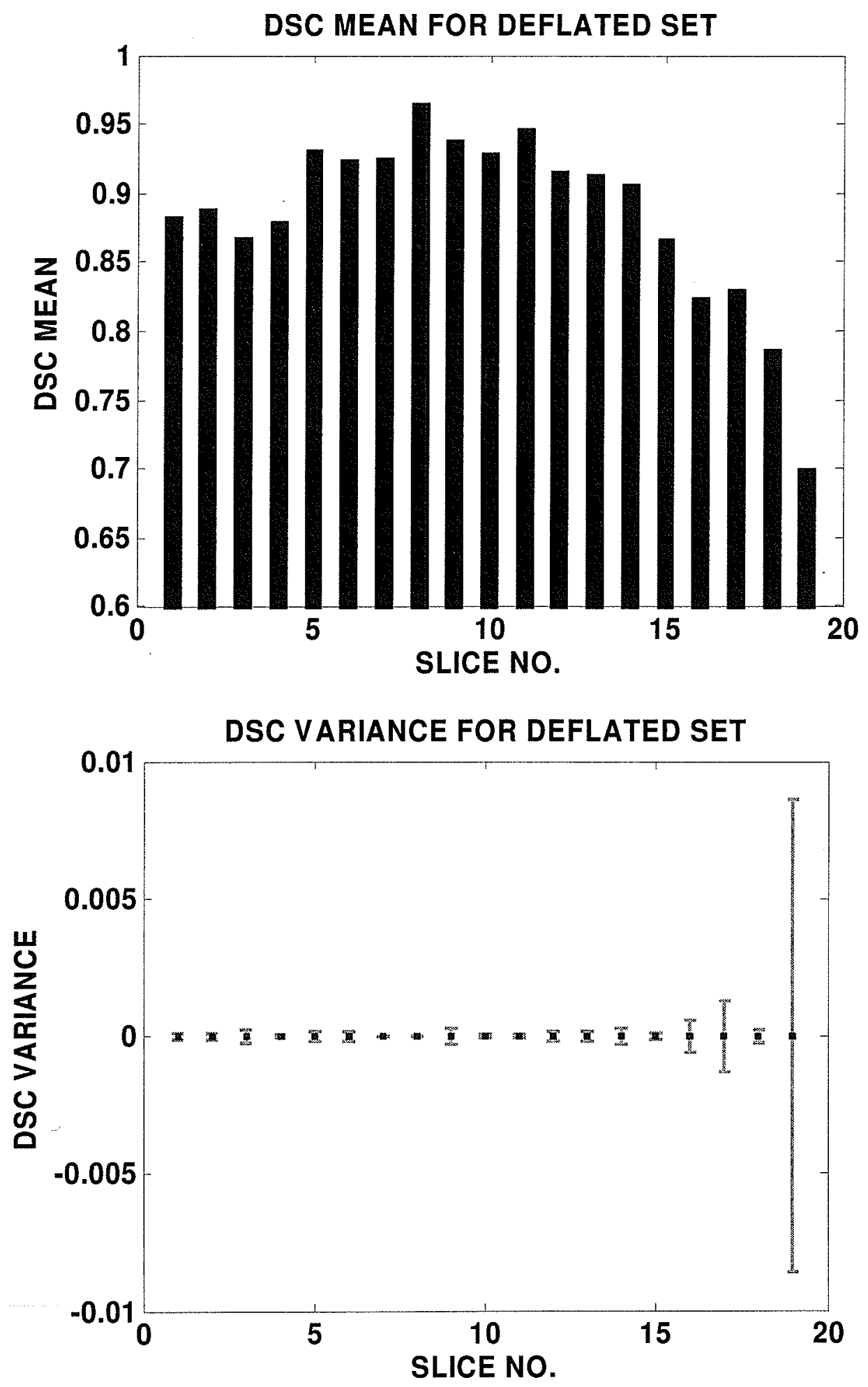

Fig. 3.4. Statistical analysis of $D S C$ for the deflated set, using mean-variance. 

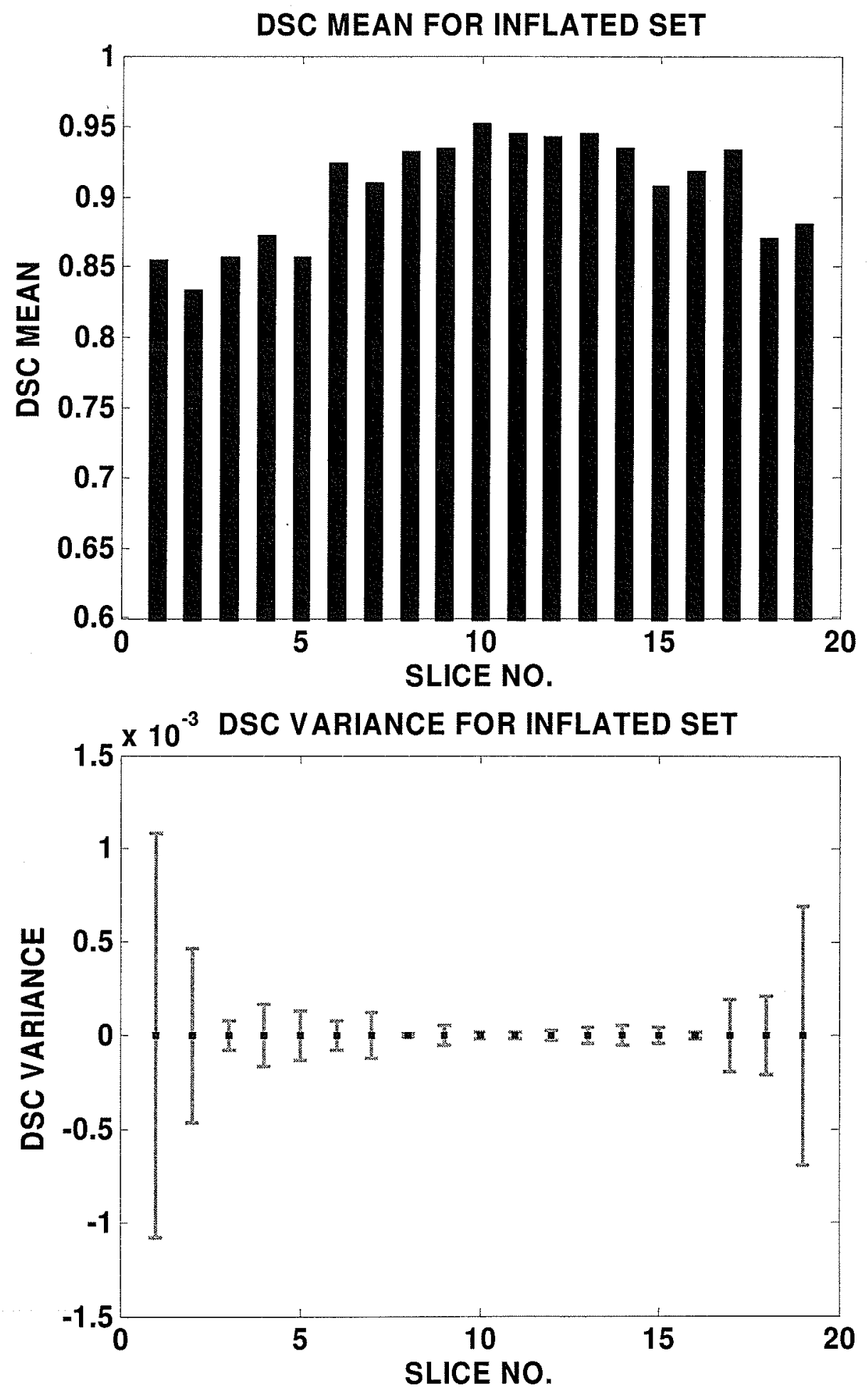

Fig. 3.5. Statistical analysis of $D S C$ for the inflated set, using mean-variance. 

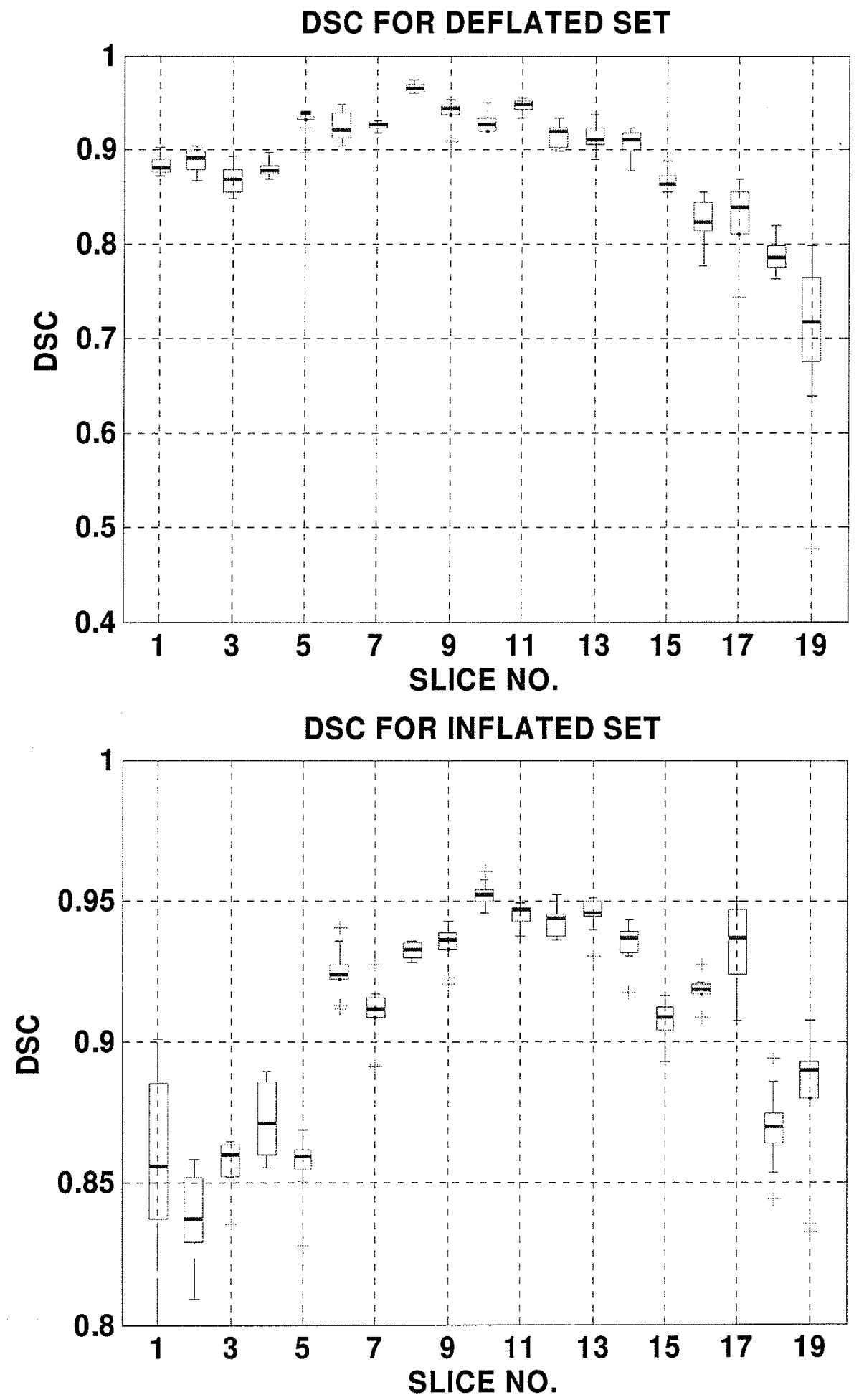

Fig. 3.6. Statistical analysis of $D S C$ using box plots, for both the deflated and inflated sets. 


\section{CHAPTER 4}

\section{Conclusions and Future Work}

\subsection{Conclusions}

Due to inter-patient variability intrinsic in biological structures, medical image analysis can be a very difficult task. Over the last decade, there has been significant interest in methods that use shape/appearance models to segment images. Using a model confines the solutions to valid instances of the structures; note that they should be modelled with a robust performance.

To date most of the prostate segmentation methods consider ultrasound and CT as the imaging modality. Recently, segmentation has been extended to include MR imaging. For example, Tsai et al. [5] applied a model-based curve evaluation technique for the segmentation of 3D prostate MRI. In addition, Freedman et al. [8] demonstrated a modelbased distribution-matching method to segment $3 \mathrm{D}$ images of prostate MRI. To the best of our knowledge, only one attempt has been published (Zwiggelaar et al. [1]), which covers the (semi-) automatic segmentation of prostate MRI. Since the corresponding training sets and the developed algorithms of the above methods are not available currently, a direct comparison between these various methods is not possible. 
In summary, we presented a novel semi-automatic method for the segmentation of the prostate in $\mathrm{T} 2$-weighted $\mathrm{MR}$ images. On this method, information from the orientation image and also four user-selected initial input points used in an empirical shape model of the prostate, which allows the boundary of prostate to be traced in $2 \mathrm{D}$.

The traced boundary is one pixel wide and can be projected back onto the original prostate images. A direct comparison with manual segmentations shows a good correlation, however, the semi-automatic approach overestimated the size of the prostate; the amount of overestimation was not consistent along the boundary. For some particular cases, such as the first and last slices, in which the variation of the gland shape is extremely large and prostate edge is more difficult to be distinguished because of the proximity of the other organs to the prostate, this approach failed to perform well.

As a semi-automatic technique, four parameters need to be selected at four different positions in the boundary of the prostate of each slice. It was shown that the developed technique was robust with respect to variations in these parameters when the result of ten times segmentation of each slice with four different input points in each trial was discussed. According to the results of statistical ranking analysis, the method's robustness regarding the four initial inputs is better for the deflated. Conversely, the method's accuracy is better for the inflated set with the minimum DSC, estimated 0.90 in average, where the same results for the deflated set is close to 0.88 . This result is promising for the applications such as prostate definition for MRSI acquisition (where the prostate boundary is used to place lipid saturation bands, as well as defining a physical boundary around tissue from which the MRS signal is acquired). Also for applications such as radiation treatment planning where the accuracy of prostate segmentation is more 
critical, the presented algorithm's accuracy is nearly as good as intra-operator accuracy (i.e. the ability of radiation oncologist to consistently segment the prostate).

The developed approach uses simple computer vision techniques in the Cartesian space of the original MRI data. Once the four initial inputs are determined, the segmentation process completes in less than 5 seconds. In the two clinical applications, we observed generally satisfactory but variable validation results.

\subsection{Future Work}

Investigating the development of a fully automatic method including the automatic estimation of the four initial points would be a beneficial extension of this work. Further research could also be done to improve the empirical shape model. Incorporating a trace modification capability to help the expert apply the necessary changes in the segmentation results is very useful.

Combining the active contour approach with the segmentation results of the middle slices in each of the volume sets is a worthwhile extension. According to the previous chapter, the outcome of this segmentation method for the middle slices in both of the clinical sets was the most accurate. On the other hand, the middle slices are the largest slices. Therefore, their extracted boundary could be used for deformable object segmentation (also known as active contours), as the initial contour which should converge to the boundary of the other smaller slices of the volume set. 


\section{BIBLIOGRAPHY}

[1] R. Zwiggelaar and Y. Zhu, "Semi-automatic segmentation of prostate," SpringerVerlag, Vol. 37, No. 3 (200), 1108-1116, 2003.

[2] R. Zwiggelaar, Y. Zhu and S. Williams, "Prostate Segmentation: a comparative study, http://www.prostate- research .org.uk/research/reports/R200218-3-will20041025.htm", 129-132, 2003.

[3] N. Venugopal, "Image Registration for Radiotherapy Applications", University of Manitoba, Winnipeg, 2004.

[4] M. Kass, A. Witkin, and D. Terzopoulos, "Active contour model," International Journal of Computer Vision, Vol.1 , No. 4, 321-331, 1987.

[5] A. Tsai, A. Yezzi, Jr., W. Wells, C. Tempany, D. Tuker, A. Fan, W.Eric Grimson and A. Willskey, "A Shape-Based Approach to the Segmentation of Medical Imagery Using Level Sets," IEEE Transactions on Medical Imaging, Vol. 22, No. 2 , February 2003.

[6] I. Dryden and K. V. Mardia, The statistical analysis of shape, Wiely, London, 1998.

[7] H. Ladek, F. Mao, Y. Wang," Prostate Segmentation from 2D Ultrasound images," In world Congress on Medical Physics and Biomedical Engineering, 2000.

[8] D. Freedman, Richard. J. Radke, Tao Zhang, Youngwon Jeong and George T.Y. Chen, "Model-Based Multi-Object Segmentation via Distribution Matching," IEEE Workshop on Articulated and Nonrigid Motion (in conjunction with IEEE CVPR), Baltimore, MD, June 2004.

[9] D. Maio and D. Maltoni," Direct Gray-Scale Minutiae Detection in Fingerprints," IEEE Transaction on Pattern Analysis and Machine Intelligence, Vol. 19, No.1, 27-40, January 1997.

[10] A.Grasselli, "On the automatic classification of fingerprints," in methodologies of Pattern Recognition, S.Watanabe (Ed.), Academic, New York, 1969.

[11] D. Maltoni, Dario Maio, Anil K. Jain and Salil Prabhakar, Handbook of Fingerprint Recognition, Springer-Verlag, 2003.

[12] A. M.Bazen and S. H.Gerez, "Systematic methods for the computation of directional fields and singular points of fingerprints", IEEE Transaction on Pattern Analysis and Machine Intelligence, Vol. 24, No.7, 905-919, 2002.

[13] P. Perona, "Orientation Diffusions," IEEE Trans. Image Processing, Vol.7, No.3, 457-467, Mar 1998.

[14] M.Kass and A.Witkin, "Analysing oriented patterns", Computer Vision, Graphics, and Image Processing, Vol. 37, No.3, 362-385, 1987. 
[15] K. Karu and A. K. Jain, "Fingerprint Classification", Pattern recognition, Vol. 29, No. 3, 389-404, 1996.

[16] M. Kawagoe and Tojo "Fingerprint Pattern classification," Pattern Recognition, Vol.17, No.3, 295-303, 1984.

[17] C. L. Wilson, G.T. Candela, and C. I. Watson, "Neural network Fingerprint Classification," Journal of Artificial Neural Networks, Vol. 1, No. 2, 203-228, 1994.

[18] R.M. Stock and C.W. Swonger, "Development and Evaluation of a Reader of Fingerprint minutiae," Cornell Aeronautical Laboratory, Technical Report CAL No. XM-2478-X-1:13-17, 1969.

[19] B.M. Mehtre, N. N. Murthy, S. Kapoor, and B. Chatterjee, "Segmentation of Fingerprint Image Using the Directional Image," Pattern Recognition, Vol. 20, No. 4, 429-435, 1987.

[20] M. J. Donahue and S. I. Rokhlin, "On the Use of Level Curves in Image Analysis," Image Understanding, Vol.31, No.5, 652-655, 1992.

[21] L. Hong, Y. Wan and A. Jain, "Fingerprint Image Enhancement: Algorithm and Performance Evaluation," IEEE Transaction on Pattern Analysis and Machine Intelligence, Vol.20, No. 8, August 1998.

[22] T. F. Cootes and C. J. Taylor," Statistical Models of Appearance for Medical Image Analysis and Computer Vision," SPIE Medical Imaging, 2001.

[23] J. Park, D. Metaxas, A.Young, and L.Axel, "Deformable models with parameter functions for cardiac motion analysis from tagged MRI data," IEEE Transactions on Medical Imaging, Vol. 5, 278-289, 1996.

[24] A.P. Petlandand and S. Sclaroff, "Closed form solutions for physically based modelling and recognition," IEEE Transactions on Pattern Analysis and Machine Intelligence, Vol. 13, No.7, 715-729, 1991

[25] U. Grenander and M. Miller, "Representation of Knowledge I Complex systems," Journal of Royal Statistical Society, Vol. B56, 249-603, 1993.

[26] C. Goodall, "Procrustes methods in the statistical analysis of shapes," Journal of the Royal Statistical Society, Vol. B53, No.2, 285-339, 1991.

[27] F. L. Bookstein, "Principal wraps: Thin-plate splines and decomposition of deformations, "IEEE Transactions on Pattern Analysis and Machine Intelligence, Vol.11, No. 6, 567-585, 1989.

[28] J. Subsol, J. P. Thirion, and N. Ayache, "A general scheme for automatically building 3D morph metric anatomical atlases: application to a skull atlas," Medical Image Analysis, Vol. 2, 37-60, 1998.

[29] K. H. Zou, S. K. Warfield and A. Bharatha, "Statistical Validation of Image Segmentation Quality Based on a Spatial Overlap Index," Acad Radiol, Vol.11, 178-189, 2004. 
[30] L. R. Dice.," Measures of the amount of ecologic between species," Ecology, Vol. 26, 297-302, 1954.

[31] J. L. Fleiss, "The measurement of interrater agreement. In: Statistical methods for rates and proportions", Second Edition, New York, NY: John Wiley \& Sons. 212$236,1981$.

[32] Jaccard $P$. The distribution of flora in the alpine zone. New Phytologist, Vol. 11, 37-50, 1912.

[33] K. H. Zou, C. M. Tempany, J. R. Fielding, S. G. Silverman., "Original smooth receiver operating characteristics curve estimation from continuous data: statistical methods for analyzing the predictive value of spiral CT of ureteral stones", Acad . Radiol, Vol. 5, 680-687, 1998.

[34] K. H. Zou, W. M. III.Wells, R. Kikinis, S. K.Warfield., "Three validation metrics for automated probabilistic image segmentation of brain tumours. Stat Med 2003.

[35] K. H. Zou, W. M. III.Wells, M. R. Kaus, R. Kikins, F. A. Jolesz, S. K. Warfield "Statistical validation of automated probabilistic fractional segmentation against composite latent expert gold standard in MR imaging of brain tumours". In:

Proceedings of fifth International Computing and Computer Assisted Interventions, Sept 25-28, 2002, Tokyo, Japan, Berlin: Springer-Verlag, 315-322

[36] G. Gerig, M. Jomier, Chakos M. Valmet, "A new validation tool for assessing and improving 3D object segmentation", In Proceeding of Fourth International Conference on Medical Imaging Computing and Computer assisted Interventions, Oct 14-17,2001, Utrecht, The Netherlands. Heidelberg: Springer, 516-523, 2001.

[37] D. P. Huttenlocher, G. A. Klauderman, W. J. Rucklidge., "Comparing images using the Hausdorf-distance", IEEE Trans Pattern Anal Machine Intel, Vol.15, 850-863.

[38] A. P. Zijdenbos, B. M. Dawant, R. A. Margolin, A. C. Palmer.," Morphometric analysis of white matter lesions in MR images: method and validation," IEEE Trans Med Imaging, Vol.13, 716-724, 1994.

[39] N. Venugopal, B. McCurdy, A. Hnatov and A. Dubey, "A feasibility study to investigate the use of thin-plate spines to account for prostate deformation, "Physics in Medicine and Biology, Vol. 50, No.12, 2871-2885, June 2005

[40] A.R. Rao and R.C Jain, "Computerized Flow Field Analysis: Oriented Texture Fields," IEEE Trans. Pattern Analysis and Machine Intelligence, Vol.14, No. 7, pp 693-709, July 1992.

[41] N. Ratha, S. Chen, and A. Jain, "Adaptive Flow Orientation Based Feature Extraction in Fingerprint Images," Pattern Recognition, Vol. 28, 1657-1672, Nov. 1995.

[42] A. K. Jain L. Hong, S. Pankanti, and R. Bolle, "An Identity-Authorization System Using Fingerprints," Patten Recognition, Vol. 9, 1365-1388, Sept. 1997.

[43] R. C. Gonzales and R. E. Woods, Digital Image Processing, Prentice Hall, Second Edition, 2002. 
[44] D. M. J. Tax, Exercise for Computer Service Praktikum AP3501 (old TN3571-P): Exploratory Data Analysis, http://www.ph.tn.tudelft.nl.

[45] M. Samiee, G. Thomas and R. Fazel-Rezai, "Semi-Automatic Prostate Segmentation of MR Images Based on Flow Orientation," IEEE Transaction on Signal Processing and Information Theory, August 2006, Vancouver.

[46] S. Di Zenzo, "A note on the Gradient of a Multi-Image," Computer Vision Graphics, and Image Processing, Vol. 33, 116-125, 1986

[47] J. Park, D. Metaxas, A. Young, and L.Axel, "Deformable models with parameter functions for cardiac motion analysis from tagged MRI data," IEEE Transactions on Medical Imaging, Vol. 5, 278-289, 1996.

[48] P. Lipson, A. L. Yullie, D. O'keeffe, J. Cavanaugh, J. Taaffe and D. Rosentahal, "Deformable templates for feature extraction from Medical Images," in $1^{\text {st }}$ European conference on computer Vision, O. Faugeras, ed., 413-417, Spring Verlag, Berlin/New York, 1990.

[49] R. Alteroviz, K. Goldberg, J. Pouliot, I-Choe Joe Hsu, Y. Kim, S. Mohyer Novoroloski and J. Kurhanewicz, "Registration of MR prostate images with biomechanical modelling and nonlinear parameter estimation," Medical Physics, Vol. 33, No. 2, 446-454, February 2006.

[50] Bajcsy and A. Kovacic, Multiresolution elastic matching," Computer Graphics and Image Processing, " Vol. 46, 1-21, 1989.

[51] Bajcsy and R. Liberson and M. Reivich, " A Computerized System for the Elastic Matching of Deformed Radiographic Images to Idealized Atlas Images, Multiresolution elastic matching," Computer Assist. Tomographic, Vol.7, 618-625, 1983.

[52] G. E. Christensen, R. D. Rabbitt, M. I. Miller," Topological Properties of Smooth Anatomic Maps, " in 14 $4^{\text {th }}$ Conference on Information Processing in Medical Imaging, France, Vol. 46, 101-112, Kluwer Academic Publishers, 1995.

[53] Ron Alteroviz and Ken Goldberg, "Registration of MR prostate images with biomechanical modelling and nonlinear parameter estimation," Medical Physics, Vol. 33(2), 446-454, 2006.

[54] Faiz M. Khan, "Treatment planning in radiation oncology", Lippincott Williams \& Wilkins, Second Edition, Philadelphia, 2006.

[55] Lester Kwock, J. Keith Smith, "Clinical Role of Proton Magnetic Resonance Spectroscopy In Oncology: Brain, Breast and Prostate Cancer, " Lancet Oncol, Vol. 7, 859-868, 2006.

[56] http://www.cancer.ca/ccs/internet/standard/0,3182,3172_14471_371299_langIden, $00 . h$ tml

[57] http://www.radiologyinfo.org/en/info.cfm?pg=mr_prostate

[58] http://www.radiologyinfo.org/en/info.cfm?pg=us-prostate\&bhcp=1 
[59] http://people.csail.mit.edu/fsegonne/research/TopologyCorrection/AIM-2005$\underline{020 . p d f}$ 